\title{
In-Depth Oral Presentations and Oral Communications
}

\section{IN-DEPTH ORAL PRESENTATIONS}

\section{SHOULDER AND ELBOW}

Massive rotator cuff tear treated with synthetic patch: long-term follow-up in a case report 15 years after surgery

G. Della Rotonda $^{1}$, R. Russo ${ }^{2}$

${ }^{1}$ Università degli Studi di Napoli Federico II (Naples, ITALY);

${ }^{2}$ Ospedale dei Pellegrini (Naples, ITALY)

Massive rotator cuff tears can not be repaired because of a large defect associated with tendon atrophy and retraction and muscle fatty degeneration.

The surgical treatment provides several options: open or arthroscopic debridment with or without subacromial decompression, arthroscopic isolated biceps tenotomy, partial repair of tendons, the use of synthetic or biologic patches, tendons transfers, hemiarthroplasty, reversed arthroplasty, arthrodesis.

Since 1986 synthetic patches were used for the treatment of rotator cuff tears as published by Ozaki who reported the short surgical experience with Marlex mesh, Teflon fabric and Teflon felt. Results on 50 shoulders treated with Teflon Felt with a mean follow-up period of 4.1 years were reported to be satisfactory in $90 \%$ of cases, but there are no published data concerning longer follow-ups. In recent years biologic patches allografts or xenografts made of purified 10 type I collagen have been increasing the suture strength and the healing of the tendons.

We report a particular case of massive rotator cuff tear treated with Marlex mesh (3 mm expanded polytetrafluoroethylene soft tissue patch), checked clinically, radiologically and with MRI control 15 years after the surgical implant.

\section{A bipolar radial head prosthesis for unreconstructable fractures of the radial head: clinical experience}

\section{F. Stacca, F. Modena, A. Celli}

Clinica Ortopedica e Traumatologica, Policlinico Modena (Modena, ITALY)

Introduction For the acute management of Mason III-IV radial head fractures, ORIF is the treatment of choice. When ORIF is not technically possible because radial head is unreconstructable, and simple radial head resection is contraindicated because of the presence of associated destabilizing lesions, radial head arthroplasty can be considered. In this retrospective review study we report our mid-term experience with a bipolar radial head prosthesis (CRF II, Tornier). Material and methods Between 2000-2007, 73 bipolar radial head prostheses were implanted consecutively at our Institution for the treatment of both acute and chronic elbow pathologies, in 16 cases the prosthesis was implanted for the acute treatment of unreconstructable radial head fractures without concurrent coronoid fractures. The original lesions were Mason III in 9 cases and Mason IV (with an associated elbow dislocation) in 7 cases. After a mean follow-up of 41.7 months, these 16 patients were assessed clinically and functionally using two standardized scores: MEPS and DASH, and radiographically. Statistical analysis was used to validate our results. Results Outcome was satisfactory in $87.5 \%$ of cases, with $75.0 \%$ of patients who recovered both the functional arcs of elbow motion. On the whole, mean ranges of motion of $116.9^{\circ}$ in flexion-extension and $159.6^{\circ}$ in pronation-supination, both greater than respective functional arcs of elbow motion. Results did not appear to be influenced in a statistically significant way by the type of original injury (Mason type III vs. IV). All elbow joints were stable, with no valgus instability, postero-lateral rotatory instability or ulno-humeral subluxation. The main complication was the development of heterotopic ossifications, which led to complete ankylosis in 2 cases and to proximal radioulnar synostosis in 2 cases. $75.0 \%$ of patients declared they were fully satisfied.

Discussion Basing on our experience, the bipolar radial head prosthesis represents a suitable option for acute treatment of unreconstructable radial head fractures, either isolated or associated with elbow dislocation. A careful surgical procedures, with repair of the Lateral-Collateral Ligament to avoid PLRI, can restore joint stability while the bone and the soft tissues heal, thus allowing early mobilization which reduces risks of post-traumatic stiffness. Further studies are required to assess results after a long-term follow-up.

\section{HAND AND WRIST}

Resection of the proximal carpal row in degenerative pathology of the radio-carpal joint

G. Taccardo, R. De Vitis, A. Militerno, S. Serpieri, F. Fanfani, F. Catalano

Complesso Integrato Columbus, Policlinico A. Gemelli, Università Cattolica Sacro Cuore (Rome, ITALY)

Objective The degenerative pathology of the radio-carpal joint, often associated to a degeneration of the intercarpal joint, is rarely primitive, more frequently it is secondary to an altered geometry of the wrist as a result of different pathological conditions:

- Fractures and nonunions of the distal radius and the carpal bones; - Osteonecrosis of the lunate and the scaphoid bone; 
- Capsulo-ligamentous lesion of wrist (chronic instability);

- Rheumatic and septic arthritis

Material and methods There was an important evolution of the surgical techniques in the treatment of this pathological condition. Today total arthrodesis of wrist is exclusively reserved to cases in which a rheumatic or septic aetiology is recognised or to cases in which other surgical procedures cannot be performed. Today the surgical procedures used for the treatment of the degenerative pathology of radio-carpal joint are: proximal row carpectomy, scaphoid excision and four-corner arthrodesis of the four angles, and the prosthesis of the radio-carpal joint.

Discussion and conclusions We expose our considerations on the revision of our case histories of radio-carpal arthrosis treated by proximal row carpectomy, with a minimum follow-up of 5 years and a middle follow-up of 10 years.

\section{Suggested readings}

1. Mulford JS, Ceulemans LJ, Nam D, Axelrod TS (2009) Proximal row carpectomy vs. four corner fusion for scapholunate (Slac) or scaphoid nonunion advanced collapse (Snac) wrists: a systematic review of outcomes. J Hand Surg Eur 34(2):256-263

2. Lundborg G, Besjakov J, Brånemark PI (2007) Osseointegrated wrist-joint prostheses: a 15 -year follow-up with focus on bony fixation. Scand J Plast Reconstr Surg Hand Surg. 41(3):130-137

3. Garcia-Elias M, Lluch A, Ferreres A, Papini-Zorli I, Rahimtoola ZO (2005) Treatment of radiocarpal degenerative osteoarthritis by radioscapholunate arthrodesis and distal scaphoidectomy. J Hand Surg Am 30(1):8-15

\section{Percutaneous treatment of scaphoid non-union}

\section{Rampoldi, D. Palombi, A. Piccioli}

Centro Traumatologico Ortopedico (Rome, ITALY)

Objective Preliminary reports indicated that selected scaphoid nonunions can be effectively treated through a closed technique. We prospectively studied a series of selected scaphoid non-union that, according to a pre-operative study, underwent percutaneous bone grafting and screw fixation. The surgical technique and the obtained outcome are the object of this study.

Material and methods Twelve selected scaphoid non-union patients ( 8 male and 4 female with a mean age of 36) underwent percutaneous bone grafting and screw fixation through a minimally invasive technique. Inclusion criteria were based on examination of standard $\mathrm{X}$-rays and MRI. Patients with stable, well aligned scaphoid nonunion without extensive sclerosis or bone resorption (grade IV and V according to Slade classification) were enrolled in the study. Surgery was performed 6-38 months from the original trauma and lasted on average $50 \mathrm{~min}$. Bone grafting was harvested with a trocar biopsy from the iliac crest in 10 cases and from the distal radius in 2 . Three different screws were used: Acutrak screws in 6 cases, Twin-Fix in 4 and Herbert screws in 2. Postoperatively the wrist was immobilized in a short-arm cast or splint by a minimum of 4 weeks to a maximum of 8 .

Results Eleven out of 12 fractures united in a mean time of 14 weeks. No complications were observed at the donor site. One fracture failed union and needed a secondary open procedure. According to the Mayo Wrist score, 9 patients had an excellent result, 2 good and 1 poor.

Discussion Scaphoid nonunions with bone loss need adeguate blood supply, stable bone fixation and bone grafting to heal. When bone resorption and sclerosis is not extensive these requirements can be satisfied by a percutaneous technique. Debridment of the fracture site and re-establishment of vascular perfusion are made by axial reaming; bone grafting is inserted in the hole produced by the reamer and stable fixation secured by the screw. In our series this technique showed excellent and good results in all patients but one.

Conclusions Minimally invasive methods have the potential to minimize soft tissue damage, reducing scarring and post-operative stiffness, and improve functional outcomes. Percutaneous treatment of selected scaphoid non-unions revealed to be an effective methods and should be considered as an alternative method to more complex open surgical procedures.

Suggested reading

1. Slade JF III, Dodds SD (2006) Minimally Invasive Management of Scaphoid Nonunions. Clin Orthop Rel Res. 445:108-119

\section{Lesion of scapho-lunate ligament with arthroscopic assistance: our experience}

\author{
N. Galvano, M. Ferruzza, A. Parlato, L. Milici, M. D’Arienzo
}

Clinica Ortopedica, Ospedale Policlinico (Palermo, ITALY)

The lesion of scapho-lunate ligament causes a chronic instability of wrist with following arthritic degeneration. These injuries now are increasing and they are related to direct trauma of the carpus, with or without wrist fractures. In our Orthopaedic Department we observed 12 ligament lesions, from February 2007 to December 2008. In 3 cases the diagnosis was made immediately, because the lesion was visible to RX, while in 9 cases the suspected lesion was confirmed by RM.

Ten cases were treated by arthroscopy, and we did the curettage of space, reduction and synthesis with a $\mathrm{K}$ wire, during the same surgical procedure. Only in 2 cases we performed synthesis with anchors through dorsal access after arthroscopy. We did a clinical and RX control at 3,6, and 12 months and in $85 \%$ of cases we observed good results evaluated with the Mayo Wrist Score. According to literature we found the best results in case of lesion repair within 6 months and the arthroscopic assistance in mandatory even for early diagnosis and better results.

\section{Ethyl-2-cyanoacrylate applied in direct contact with the epineurium does not impair nerve regeneration taking place in a nerve-guide of new design}

\section{A. Merolli, L. Rocchi, A. Morini, L. Mingarelli, F. Catalano}

Clinica Ortopedica, Università Cattolica (Rome, ITALY)

Objective Stitch suture is still the most recommended method to hold a nerve-guide. However, stitch suture is a well known cause of local inflammatory response. Glues of several kinds have been proposed as an alternative but they are not easy to be used in a real surgical setting. In 2006 we developed a new concept of nerve-guide termed "NeuroBox" which is double-halved, not-degradable and rigid, and allows the use of cyanoacrylic glues [1]. In this study, we analyzed histologically the nerve-glue interface.

Material and methods Wistar rats were used as animal model. In group 1, animals were implanted a NeuroBox to promote the regeneration of an experimentally produced $4 \mathrm{~mm}$ gap in the sciatic nerve. In group 2, the gap was left without repair ("sham-operated" group). 
Group 3 was assembled by harvesting 10 contralateral intact nerves to document the normal anatomy. Semi-thin sections for visible light microscopy and ultra-thin sections for Transmission Electron Microscopy were analyzed.

Results Results showed that application of ethyl-2-cyanoacrylate directly to the epineurium produced neither significative insult to the underlining nerve fibers nor impaired nerve regeneration. No regeneration occurred in the "sham-operated" group.

Discussion As a group of rapidly polymerizing adhesives, cyanoacrylates have shown promising results in nerve surgery [2] but their actual use in a true surgical setting may bear a lot of technical difficulties; this may explain, in part, early negative recommendations [3]. Conclusions In the present work, we found that ethyl-2-cyanoacrylate was easily applied in the peculiar construct of the NeuroBox and nerve regeneration was not affected by the presence of the acrylic glue all around the epineural sheath of the glueing regions. In particular, no alterations were found in the morphology of axons and Schwann's cells and these results confirm that ethyl-2-cyanoacrylate can be used in direct contact with the nerve.

\section{References}

1. Merolli A et al. (2009) In vivo regeneration of rat sciatic nerve in a double-halved stitch-less guide: a pilot-study. Microsurgery 29(4):310-318

2. Rickett T et al. (2009) Ethyl-cyanoacrylate is acutely nontoxic and provides sufficient bond strength for anastomosis of peripheral nerves. J Biomed Mater Res 90(3):750-754

3. Wieken K et al. (2003) Nerve anastomosis with glue: comparative histologic study of fibrin and cyanoacrylate glue. J Reconstr Microsurg 19(1):17-20

\section{FOOT AND ANKLE}

\section{Arthroplasty of the ankle: our experience}

\section{Spinella, M. Ferlazzo}

Clinical Institute of Orthopaedics and Traumatology Care (Messina, ITALY)

Introduction The ankle is a pulley with a single movement that affects the movement of the leg in relation to the foot on sagittal floor. It is subjected, like all joints, to traumatic events sprains, fractures (fracturesdislocation) infiammatory diseases (rheumatoid arthritis, psoriasic arthritis, uric acid, etc.), degeneration resulting in osteoarthritis (secondary osteoarthritis) and degenerative disorders (primary osteoarthritis). Material and methods Since January 2008 to May 2010, 15 patients have been treated, 8 male and 7 female, aged between 47 years and 72; three cases were primary osteoarthritis and 12 cases secondary. All patients had a limited excursion (at least half) with persistent pain also at rest. According to international protocols, patients, who underwent surgery aged more than 47 years, they were non-obese, without severe osteoarthritis of the hip, knee, subtalar and average foot ipsilateral, without serious circulatory or neurological disorders, and had possibly a misalignment of the ankle superior to 15 degrees in the frontal plane and with a good bone-stock.

Hints of surgical technique We always implanted a biological prosthesis with mobile insert. We performed a frontal surgical approach with removal of impingement and osteophytes, the maximum resection of the tibia did not exceed 10 and $4 \mathrm{~mm}$ as astragalus, it was necessary to assail defects not stabilize the ankle. Surgery was performed in regional anesthesia (Bi-Block); we maintained the discharge into the plaster splint for 2 weeks and then applied knee pinstriped cargo for 2 weeks before beginning rehabilitation. Followup was 24 months.

Results During the process of results evaluation we considered 2 main parameters: (1) pain at rest and after exercise; (2) articular parameters. In all cases we observed a significant improvement with a net reduction of the joint pain both on walking and at rest.

Conclusions Although our brief experience and even a short follow-up, our results led us to consider prosthetic replacement of the ankle as the primary indication in patients who face the above mentioned parameters. Anyway, in our opinion, this challenging surgery should be proposed to young and active patients, who do not accept arthrodesis

\section{Metatarso-phalangeal and interphalangeal hallux valgus: surgical treatment by SERI-Akin combined osteotomy}

S. Giannini, C. Faldini, S. Pagkrati, D. Leonetti, M. Nanni, F. Acri, M.T. Miscione

Istituto Ortopedico Rizzoli (Bologna, ITALY)

Objective Combined metatarso-phalangeal and inter-phalangeal deformity represents about $1 \%$ of hallux valgus deformity, and its treatment remains a debated topic, because a single osteotomy does not entirely correct the deformity and double osteotomies are needed. The aim of this study is to review the results of 50 consecutive combined metatarso-phalangeal and inter-phalangeal hallux valgus treated by Akin proximal phalangeal osteotomy and SERI minimally invasive distal metatarsal osteotomy.

Material and methods Fifty feet in 27 patients, aged between 18 and 75 years (mean 42 years) affected by symptomatic hallux valgus without arthritis were included. Two $1-\mathrm{cm}$ medial incisions were performed at the metatarsal neck and at proximal phalanx. Then SERI osteotomy was performed to correct metatarso-phalangeal deformity and Akin osteotomy was performed to correct interphalangeal deformity. Both osteotomies were fixed with a single K-wire. A gauze bandage of the forefoot was applied and immediate weight-bearing on hindfoot was allowed. K-wire was removed after 4 weeks. All patients were checked at a mean 4-year follow-up.

Results All osteotomies healed. Delayed union of metatarsal osteotomy was observed in 1 foot. Slight stiffness was observed in 2 feet. Mean AOFAS score was $47 \pm 15$ preoperatively and $91 \pm 9$ at last follow-up. Radiographic findings revealed a significant improvement $(p<0.005)$ of interphalangeal-angle (pre-op $17.5^{\circ}$, post-op $5.1^{\circ}$ ), hallux-valgus-angle (pre-op $30.1^{\circ}$, post-op $12.2^{\circ}$ ), inter-metatarsal-angle (pre-op $13.4^{\circ}$, postop $7.1^{\circ}$ ), distal-metatarsal-articular-angle (pre-op $20.1^{\circ}$, post-op $8.2^{\circ}$ ).

Conclusions The combined SERI-Akin double osteotomy was an useful procedure for correction of complex hallux valgus deformity. Clinical and radiographic findings showed an adequate correction of all parameters of the deformity.

\section{Surgical treatment of high grade hallux rigidus by arthroplasty using bioreabsorbable implant: a review of 42 operated cases}

S. Giannini, C. Faldini, D. Leonetti, M. Nanni, F. Acri, M.T. Miscione

Istituto Ortopedico Rizzoli (Bologna, ITALY)

Objective High grade hallux rigidus is a forefoot deformity characterized by a limitation of dorsiflexion of the first toe associated with 
pain, and severe damage of the first metatarsophalangeal joint. Most authors recommended resection arthroplasty or arthrodesis of the first metatarsophalangeal joint. The aim of this study was to present the results of our series of 42 consecutive cases of severe hallux rigidus treated by resection of the first metatarsal head and implant of a poly D-L lactic (PDLLA) bioreabsorbable spacer to promote the interposition of fibrous tissue to preserve the range of motion of the joint. Material and methods Forty-two feet in 27 patients affected by high grade hallux rigidus were included in the study. Surgical treatment consisted of resection of the first metatarsal head and positioning of a poly D-L lactic acid (PDLLA) bioreabsorbable implant. Post-operative care consisted in gauze bandage of the forefoot, and immediate weight-bearing with talus shoes for 3 weeks. All patients were clinically and radiographically evaluated preoperatively and checked at a mean 5-year (range 3-7) follow-up.

Results No intraoperative complication occurred. No sinus formation were observed. All implants resulted well tolerated by the patients. Mean AOFAS score was $42 \pm 14$ preoperatively and $81 \pm 9$ postoperatively. Mean preoperative metatarsophalangeal joint ROM was $27 \pm 17$ degrees and mean postoperative metatarsophalangeal joint ROM was $75 \pm 8$ degrees.

Conclusions First metatarsophalangeal joint arthroplasty using bioreabsorbable implant demonstrated to be an effective surgical option for treatment of high grade hallux rigidus, thank to its ability to promote fibrous tissue formation during its reabsorption.

\section{SPINE}

\section{C1-C2 fixation for treatment of traumatic upper cervical spine injuries}

\author{
L. Proietti, E. Pola, L. Scaramuzzo, G.R. Schirò, S. Sessa, \\ C.A. Logroscino
}

Spine Surgery Division, Department of Orthopaedic Science and Traumatology, Catholic University (Rome, ITALY)

Objective Traumatic upper cervical spine injuries are, still nowadays, a complex pathology. Complexity is due to the peculiarity of the occipito-cervical and atlanto-axial junctions and to the possible vascular complications related to the surgical treatment. Mechanical instability in the cervical spine is characterized by important pain and risk for neurological involvement, therefore the achievement of a good mechanical stability, in the treatment of these injuries is fundamental. Aim of the study was to evaluate clinical and functional results of patients affected by fractures or pseudoarthrosis of the upper cervical spine treated by $\mathrm{C} 1-\mathrm{C} 2$ fixation.

Material and methods From August 2006 to August 2009 in the Spine Division of the Department of Orthopaedic and Traumatology of Catholic University, 10 patients ( 8 males and 2 females) with a mean age of 43 years (range 18-86 years) underwent the index procedure. Seven patients presented a pseudoarthrosis of $\mathrm{C} 2$ and 3 a complex fracture of $\mathrm{C} 1-\mathrm{C} 2$. All patients were evaluated post-operatively by antero-posterior and lateral X-ray at regular time intervals, and by $\mathrm{CT}$ of the treated levels to evaluate the screws positioning. Clinical outcome was evaluated by periodically administration of SF-36 questionnaire, Oswestry Disability Index and VAS Score.
Results All patients showed at 20 months median follow-up (min. 6, max. 36 months) a fast and progressive pain improvement (VAS score 2.2 at 20 months medium follow-up), a fast complete functional recovery (ODI 11.4\% 20 months median follow-up) and a consequent quality of life improvement (SF-36 PCS $89.7 \%$ e MCS $85.9 \%$ at 20 months median follow-up). Radiographic evaluation showed a good screws positioning and the achievement of an optimal mechanical stability. In one patient there was an intra-operative complication: a great bleeding from the $\mathrm{C} 1-\mathrm{C} 2$ venous plexus, that prevented the insertion of screws in the $\mathrm{C} 2$ pedicles, these were so inserted with a translaminar technique. There were no post-operative complications and no hardware failure.

Discussion Harms C1-C2 fixation is a valid and safe technique for the treatment of upper cervical spine injuries. Advantages of this surgical technique, with respect to the traditional occipito-cervical fusion, are less associated comorbidities and a greater residual cervical spine motility. It allows, in severe lesions, to avoid the permanent use of brace, poorly tolerated, particularly from the elderly. In our opinion, this surgical technique, even though complex, presents a good repeatability, a reasonable versatility and a mechanical stability comparable to the one observed in traditional occipito-cervical fusion.

\section{Sagittal segmental alignment of the cervical spine following single level anterior interbody fusion is a predictor of adjacent-level degeneration}

\author{
C. Faldini, D. Leonetti, M.T. Miscione, M. Nanni, F. Acri, \\ S. Pagkrati, S. Giannini
}

Istituto Ortopedico Rizzoli (Bologna, ITALY)

Objective The Cloward anterior interbody fusion is commonly performed for cervical disc herniation or spondylosis. In follow-up studies, various authors noted clinically relevant adjacent-level degeneration. However, factors associated with adjacent-level degeneration are not well known. We investigated whether the postoperative sagittal segmental alignment of the fused vertebrae could be used as a predictor of adjacent-level degeneration.

Material and methods We retrospectively studied 107 patients, aged 35-55 years, with one-level cervical disc disease between $\mathrm{C} 4$ and $\mathrm{C} 7$ operated by discectomy and one-level anterior cervical fusion according to the Cloward procedure. In standard radiographs of the cervical spine in lateral view, the alignment of the involved intervertebral space (sagittal segmental alignment, SSA) and the sagittal alignment of the cervical spine SACS) were measured and the adjacent-level degeneration was assessed using the Kellgren and Lawrence criteria. Basing on SSA, patients were divided into 2 groups: group A with SSA $<0^{\circ}$, neutral or kyphotic (41 patients), group B with SSA $>0^{\circ}$, lordotic (66 patients). The minimum follow-up was 10 years.

Results Preoperatively, mean SSA was $0.6 \pm 4$ and mean SACS was $17 \pm 9$. At last follow-up, the mean SSA was $-2.8 \pm 2$ in group A and $4.8 \pm 4$ in group B, whereas mean SACS was $14 \pm 6$ in group A and $23.5 \pm 7$ in group B. Adjacent-level degeneration was present in $60 \%$ of cases in group A and in $27 \%$ of cases in group B $(p<0.05)$. Conclusions The Cloward anterior interbody fusion is an effective technique for the treatment of cervical disc herniation or spondylosis. To prevent adjacent-level degeneration, we recommend establishing a lordotic sagittal segmental alignment when anterior interbody fusion of the cervical spine is indicated. 


\section{Treatment of thoraco-lumbar curves in adolescents affected by idiopathic scoliosis with a PASB: assessment of results according to the SRS committee}

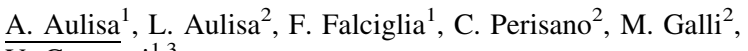 \\ V. Guzzanti ${ }^{1,3}$ \\ ${ }^{1}$ Traumatology Department, Children's Hospital Bambino Gesù \\ (Rome, ITALY); \\ ${ }^{2}$ Department of Orthopaedics, A. Gemelli Hospital, Università \\ Cattolica del Sacro Cuore (Rome, ITALY); \\ ${ }^{3}$ University of Cassino (Cassino-FR, ITALY)
}

Objective The effectiveness of conservative treatment of scoliosis is controversial. Some studies suggest that brace is effective in stopping curve progression, whilst others did not report such an effect. The purpose of the present study was to evaluate the effectiveness of Progressive Action Short Brace (PASB) in the correction of thoracolumbar curves, in agreement with the Scoliosis Research Society (SRS) Committee on Bracing and Nonoperative Management Standardisation Criteria.

Material and methods Fifty adolescent females (mean age: $11.8 \pm 0.5$ years) with thoraco-lumbar curve and a pre-treatment Risser score ranging from 0 to 2 have been enrolled. The minimum duration of follow-up was 24 months (mean: $55.4 \pm 44.5$ months). Antero-posterior radiographs were used to estimate the curve magnitude (CM) and the torsion of the apical vertebra (TA) at 5 time points: beginning of treatment $(\mathrm{t} 1), 1$ year after the beginning of treatment $(\mathrm{t} 2)$, intermediate time between $\mathrm{t} 1$ and $\mathrm{t} 4(\mathrm{t} 3)$, end of weaning (t4), 2-year minimum follow-up from $\mathrm{t} 4(\mathrm{t} 5)$. Three situations were distinguished: curve correction, curve stabilisation and curve progression. The Kruskal-Wallis and Spearman Rank Correlation tests have been used as statistical tests.

Results CM mean value was $29.30 \pm 5.16 \mathrm{SD}$ at $\mathrm{t} 1$ and $14.67 \pm 7.65$ $\mathrm{SD}$ at $\mathrm{t} 5$. TA was $12.70 \pm 6.14 \mathrm{SD}$ at $\mathrm{t} 1$ and $8.95 \pm 5.82$ at $\mathrm{t} 5$. The variation between measures of Cobb and Perdriolle degrees at $t 1, t 2$, $\mathrm{t} 3, \mathrm{t} 4$, $\mathrm{t} 5$ and between $\mathrm{CM}$ t5-t 1 and TA t5-t1 were significantly different. Curve correction was accomplished in $94 \%$ of patients, whereas a curve stabilisation was obtained in $6 \%$ of patients.

Conclusions The PASB, due to its peculiar biomechanical action on vertebral modelling, is highly effective in correcting thoraco-lumbar curves.

\section{Evaluation of conservative scoliosis treatment compliance}

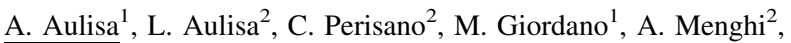 \\ V. Guzzanti ${ }^{1,3}$
}

${ }^{1}$ Traumatology Department, Children's Hospital Bambino Gesù, Institute of Scientific Research (Rome, ITALY);

${ }^{2}$ Department of Orthopaedics, A. Gemelli Hospital, Università

Cattolica del Sacro Cuore (Rome, ITALY);

${ }^{3}$ University of Cassino (Cassino-FR, ITALY)

Objective The main purpose of this study is to evaluate orthosis treatment compliance of patients affected by adolescent idiopathic scoliosis (AIS).

Material and methods We examined 106 patients (96 females, 10 males) affected by AIS. The patients were treated with different types of orthosis (Lionese, Milwaukee, P.A.S.B., Lionese + P.A.S.B., Milwaukee + P.A.S.B., Milwaukee + Lionese). In order to evaluate the compliance and the final results related to the patient's behavior, we considered the followings: the patient's compliance as related to bracing method, sex, trend of the spine curve and age, the degree of scoliosis and the duration of treatment.

Results Our study indicated a greater number of highly compliant patients $(n=66)$ than incompliant $(n=40)$. For the incompliant group, 52.5\% had not worn the brace for long time (more than 3 months), and the others only wore the brace in the summer or at school. Inside the incompliant group, $50 \%$ had been treated with the Milwaukee brace. It was also noticed that in mixed-brace treatments, the Milwaukee brace raised the percentage of incompliance. For the compliant group, $47 \%$ had been treated only with the P.A.S.B. brace and $6 \%$ with a mixed-brace treatment, including P.A.S.B. $28.3 \%$ of the patients had ceased the treatment before the scheduled date. Patients who terminated the treatment at an age of more than 17 years had a higher degree of improvement $(10.81 \%)$ compared with an age lower than 17 years $\left(9.38^{\circ} \mathrm{Cobb}\right)$. When the brace was discarded, patients who did not complete the treatment course (more than $50 \%$ wore P.A.S.B.) showed a slightly greater improvement than those who completed the treatment.

Conclusions The study indicated that brace type, patient compliance, and completion of the treatment until skeletal maturity are very important for patient outcome in the improvement of AIS. Patient compliance depends on the orthosis type and not on the sex. The compliance of Milwaukee brace is lower than the P.A.S.B., because the Milwaukee brace is more visible and has the chin support, while the P.A.S.B. fits better and is less visible. Treatment incompliance is caused by several factors, including psychological elements and radiological improvement of the spine curve that the patient mistakenly interprets as full recovery.

\section{KNEE}

Axial femoro-tibial alignment and rotation of the tibial component in total knee arthroplasty

\author{
G. Cinotti, F.R. Ripani, E. Ferrari, A. Della Rocca, W. Salustri
}

Orthopaedic Clinic, University of Rome La Sapienza (Rome, ITALY)

Introduction Total knee arthroplasty (TKA) allows to achieve a substantial pain relief and functional recovery in the vast majority of the operated patients. However, in most of the series a definite number of patients are not entirely satisfied with the surgical outcome. While in few cases the postoperative radiographs clearly show the cause of failure, in the remaining cases a definite cause of the poor outcome is not easily identified. In the latter group, once infection has been ruled out, an inadequate ligamentous balancing and/or a poor patellar tracking may be the cause of the poor result. Aim of this study was to assess the correct positioning of the tibial component with respect to the epicondylar axis and the morphologic variations of the meta-epiphyseal region of the proximal tibia.

Material and methods Axial CT scans of 50 young-adults (33 males and 27 females) were analysed. Imaging studies showing degenerative changes of the knee joint were excluded from the study. Axial CT scans were assessed at the epicondylar level, and $10 \mathrm{~mm}$ below the joint space (tibial cut level). The proximal portion of the tibial apophysis (TA) was divided in three portions (medial, central, lateral). The angle between the anterior projection of a line perpendicular to the middle of the epicondylar axis and a line connecting the latter with the medial $1 / 3$ of the TA was assessed.

Results and conclusions Our results showed that the anterior projection of a line perpendicular to the middle of the epicondylar axis was internally rotated compared to the medial $1 / 3$ of the TA in most 
of the subjects. As a result, if the epicondylar axis is the only anatomical landmark used, the tibial component may result internally rotated with respect to the patellar tendon and a poor patellar tracking may occur. On the other hand, if epicondylar axis is not taken into account, a mismatch between femoral and tibial rotation may take place, leading to early polyethylene wear. Our results suggest that at least both anatomical landmarks should be considered for a proper alignment of the tibial component in the axial plane.

\section{Expression of NGF and TrkA receptor in ACL samples from patients affected by knee osteoarthritis}

\author{
M. Surace, A. Fagetti, D. Marcolli, D. Prestamburgo, P. Cherubino
}

Dipartimento di Scienze Chirurgiche Ricostruttive e Tecnologie Avanzate (Varese, ITALY)

The presence of NGF and TrkA receptor have been demonstrated in peri-articular tissues and lumbar inter-apophyseal joints in patients affected by chronic lumbar pain.

Aim of the study is to demonstrate the presence of neurotrofin and its receptor in ACL samples hatched during total knee arthroplasty surgery in patients affected by gonarthrosis.

We recruited 15 patients affected by gonarthrosis (age: 65 years or older); 5 male and 10 females.

All specimens were fixed in formalin, dehydrated and enclosed in paraffin. From each specimen 4 slides were obtained. Two slides were employed for the search of NGF, one was treated with specific antibodies and marked with FITC (Fluorescein Isothiocyanate Conjugated), the last slide was for control purposes and was exposed to FITC, but without prior exposition to the specific antibody. The same procedure was repeated to obtain two more slides in order to repeat the search for TrkA, with specific antibodies. All the slides were finally studied on a fluoromicroscope.

The slides analysis demonstrated the presence of the neurotrofin and of the TrkA receptor. The immunohistochemical reaction showed a fluorescence in 13 of 15 slides prepared with both the antibodies. No fluorescence was displayed in the control cases. In one sample no fluorescence was found, with no evidence of NGF and its receptor; light microscopy did not reveal any inflammatory reaction in the sampled tissues. In another case, also negative at the immunohistochemical reaction, only few inflammatory cells were found in the specimen.

The presence of the NGF and its receptor is constantly associated with a chronic inflammatory aspect, the two samples that resulted negative for NGF/TrkA expression did not show any inflammatory cells.

NGF could concur in the regulation of the condrocytes and fibroblasts metabolism. It is possible that the NGF is involved in the evolution of arthritis.

\section{New nanostructured biomimetic scaffold for the treatment of osteochondral defects: pilot clinical study at 3-year follow-up}

E. Kon $^{1}$, G. Filardo ${ }^{1}$, A. Di Martino ${ }^{1}$, S. Patella ${ }^{1}$, M. Delcogliano ${ }^{2}$, S. Zaffagnini ${ }^{1}$, L. D'orazio ${ }^{1}$, B. Di Matteo ${ }^{1}$, M. Marcacci ${ }^{1}$

${ }^{1}$ Laboratorio di Biomeccanica, Clinica Ortopedica e Traumatologica III, Istituto Ortopedico Rizzoli (Bologna, ITALY);

${ }^{2}$ Ospedale S. Carlo di Nancy (Rome, ITALY)
Introduction The ideal osteochondral graft should be an off-the-shelf product; this is the research direction and the explanation for the new biomaterials recently proposed to repair osteochondral defect inducing an in situ cartilage regeneration starting from the time of the implantation into the defect site. For the clinical pilot study, a newly developed nanostructured biomimetic scaffold was used to treat chondral and osteochondral lesions of the knee; its safety and manageability, as much as the surgical procedure reproducibility and the clinical outcome, were evaluated in order to test its intrinsic potential without any cell colture aid.

Material and methods A new osteochondral scaffold was obtained by enucleating equine collagen type 1 fibrils with hydroxyapatite nanoparticles in 3 different layers with 3 different gradient ratios at physiological conditions. Thirty patients ( 9 female, 21 male, mean age 29.3 years) affected by either chondral or osteochondral lesions of the knee ( 8 medial femoral condyles, 5 lateral femoral condyles, 12 patellae, 8 femoral throcleas) underwent the scaffold implantation from January to July 2007. The sizes of the lesions were in between 2 and 6 squared $\mathrm{cm}$. All patients and their clinical outcome were analyzed prospectively at $6,12,24$ and 36 months using the Cartilage standard Evaluation Form as proposed by ICRS and an high resolution MRI.

Results We observed a statistically significant scores improvement and function recovery comparing the pre-operative to the follow-up parameters evaluated. Moreover, we noticed a better improvement from 12 to 24-month follow-up while the good results gained at 2 -year were confirmed at 3-year follow-up evaluation. The MOCART scoring scale was used to analyze the MRIs. In $80 \%$ of cases we obtained a complete filling of the cartilage defect and in some patients we even appreciated articular surface congruency. In this series we report 1 failure followed by a re-operation with a different technique. Conclusions This new minimally invasive one-step surgical approach to osteochondral defects seems to be an easy and effective procedure.

\section{Scanty integration of osteochondral allografts cryopreserved at low temperatures with dimethyl sulfoxide. An experimental study in sheep}

\section{U.G. Longo ${ }^{1}$, P. Rocco ${ }^{1}$, S. Campi ${ }^{1}$, N. Maffulli ${ }^{2}$, F. Forriol ${ }^{3}$}

${ }^{1}$ Department of Orthopaedic and Trauma Surgery, Campus

Bio-Medico University (Rome, ITALY);

${ }^{2}$ Centre for Sports and Exercise Medicine, Queen Mary University of London, Barts; The London School of Medicine and Dentistry Mile End Hospital (London, UK)

${ }^{3}$ Research Unit FREMAP Hospital (Madrid, SPAIN)

The limited availability of osteochondral allografts compared to clinical needs require economical, and medical efforts to improve the actual knowledge in the field of preservation of osteochondral allografts. Our null hypothesis was no differences in the Osteoarthritis Research Society International, International Cartilage Repair Society, Mankin, and modified Wakitani scores among 6 groups of osteochondral transplantation using 6 different protocols of allograft storage. Thirty-six adult sheep were randomized to 6 groups of allograft osteochondral transplantation. Six osteochondral cylinders were stored for 6 weeks at $-80^{\circ} \mathrm{C} ; 6$ at $-80^{\circ} \mathrm{C}$ with $10 \%$ dimethyl sulfoxide (DMSO); 6 at $-80^{\circ} \mathrm{C}$ with $10 \%$ DMSO for $90 \mathrm{~min} ; 6$ at $-186^{\circ} \mathrm{C}$; 6 at $-186^{\circ} \mathrm{C}$ with $10 \%$ DMSO; 6 at $-186^{\circ} \mathrm{C}$ for $90 \mathrm{~min}$. After transplantation, all animals were euthanized at 6 months. Harvested specimens underwent gross morphologic and histological evaluation. We found no statistically significant differences when comparing the gross cartilage morphology of each group $(p>0.05)$. No significant difference were found among the six groups, as evaluated with the Osteoarthritis Research Society International, 
International Cartilage Repair Society, Mankin, and modified Wakitani scores. The Spearman's rho was respectively 0.629 between Mankin and OARSI $(p=0.001), 0.645$ between modified Wakitani and OARSI $(p=0.001)$. The Spearman's rho was 0.391 between Mankin and modified Wakitani $(p=0.019)$.

In conclusion, the cryopreservation protocols adopted in the present study provided scanty integration in an in vivo sheep model of osteochondral allograft transplantation. The Mankin and OARSI scores and the modified Wakitani and OARSI scores showed a good correlation grade. The Mankin and modified Wakitani scores showed a fair correlation grade. On the basis of our results, we discourage the application of the storage methods used in the present study for preservation of osteochondral samples in clinical practice.

\section{Suture of meniscal tears in the avascular zone enforced by a collagen I/III membrane: clinical experience over 6 years and animal experiments}

\author{
$\underline{\text { R. P. Jakob }}{ }^{1}$, M. Jacobi ${ }^{1}$, D. Nesic ${ }^{2}$, P. Mainil ${ }^{2}$ \\ ${ }^{1}$ (Freiburg, SWITZERLAND); \\ ${ }^{2}$ (Bern, SWITZERLAND)
}

Introduction Due to the limited healing potential of meniscal tissue different efforts have been done to enhance healing of sutured meniscus.

Technique and material The meniscus wrap technique is based on the experience with a technique developed by the senior author (RPJ) and firstly applied in 2003. Wrapping the meniscus with a collagen I/III matrix might create some kind of bioreactor, guiding cell ingrowth and improving suture stability. Thirty patients with tears in the red-white or white-white zone, complex tears, delayed traumatic tears with degenerative aspects, or repeat sutures, were treated with this technique.

Results After a mean follow-up of 2.5 years (range 1-5) three patients had a symptomatic failure (10\%). In two of them partial meniscectomy was performed and in the third patient (a 20-year-old female with a second suture of a bucket-handle tear) a third suture combined with an unloading osteotomy was performed, which ultimately led to clinical meniscal healing. All other 27 cases (90\%) were asymptomatic. Additionally, the following complications were noted: arthrofibrosis requiring mobilization under anaesthesia (one patient), saphenous nerve entrapment necessitating revision (one patient), and ACL rerupture after reconstruction and a new trauma, with the meniscus remaining intact (one patient). Results of an animal experimental study (goats) shall be reported.

Conclusions This repair enhancement technique seems to improve the chances of healing, even in unfavourable conditions. Although the evaluation did not include a second-look arthroscopy, $90 \%$ of patients remained asymptomatic after a mean follow-up of 2.5 years. Similar to fascia sheath coverage, this technique has the disadvantage of being technically demanding and time-consuming.

\section{TRAUMATOLOGY}

\section{Last generation intramedullary nailing system for long bone fractures}

\section{F. Biggi, F. Dalla Vestra, S. Di Fabio, T. Pagliara, C. Salfi}

Orthopaedics and Traumatology Department, San Martino Hospital (Belluno, ITALY)
Objective Reamed locked intramedullary nailing is the current gold standard for tibial diaphyseal fractures [1,2]: early reduction and stabilization, utilizing closed technique, allow better recovery and results in terms of bone healing and function.

Material and methods Forty-two consecutive patients (16 female and 26 male) with tibial shaft fractures (33 closed fracture; 9 open fracture) treated at our Institution with anatomical intramedullary locking nail system (Zimmer Natural Nail) from January 2009 to December 2009 were retrospectively reviewed. The time of healing, complications, knee range of motion and functional capacity were evaluated at minimum of 6 months from surgery.

Results Treatment with reamed intramedullary nail led to union in all cases. Four patients sustained mild complications: one distal screw mobilization, one distal screw breakage, one superficial infection healed with antibiotics therapy (Type III B fracture) and one case of knee pain with full range of motion. Three fractures healed with almost 5 degree of angulations. A distal third fractures was found to be associated with an increased incidence of malalignment.

Discussion The Anatomical Locking Nail System provide a safe and accurate treatment for tibial fractures with extension to proximal and distal metaphyseal area, independently by the degree of comminution and number of fragments because the multiple locking screw fixation in different planes. Technique and instruments are able to allow a reproducible result for trauma surgeons facing this type of injuries.

\section{References}

1. Court-Brown C, McQueen M, Tornetta P (2006) Intramedullary nailing. In: Trauma. Lippincot William \& Wilkins, Philadelphia, pp 440-451

2. Larsen LB, Madsen JE, Høiness PR, Øvre S (2004) Should insertion of intramedullary nails for tibial fractures be with or without reaming? A prospective, randomized study with 3.8 years' follow-up. J Orthop Trauma 18:144-149

\section{Three- and four-part humeral head fractures in 3-4 ASA status patients treated with closed reduction and percutaneous pinning (MIROS System)}

\author{
M. Tangari $^{1}$, S. Carbone ${ }^{3}$, S. Gumina ${ }^{3}$, M. Gallo ${ }^{2}$, A. Campi ${ }^{2}$ \\ ${ }^{1}$ Hand Surgery, ${ }^{2}$ Orthopaedics and Traumatology, San Giovanni- \\ Addolorata Hospital (Rome, ITALY), ${ }^{3}$ Orthopaedic Clinic, \\ University of Rome La Sapienza (Rome, ITALY)
}

Introduction Closed reduction and percutaneous pinning for displaced humeral head fractures offer advantages versus open techniques since they limit the exposure, thus minimizing surgical trauma and reducing the risk of necrosis. The aim of this study is to evaluate results of three- and four-part humeral head fractures in 3-4 ASA (American Society of Anesthesiologists) status patients treated with closed reduction and percutaneous pinning.

Material and methods From 2007 to 2009, 26 patients mean aged 78 years (range: 70-87) were enrolled for this study. Criteria of inclusion were three- or four-part displaced humeral head fracture (fractures classified radiographically following Neer's system), ASA status of 3 or 4 (open reduction highly non recommended) and absence of vascular or nervous deficits. All patients were treated with the MIROS (Minimally Invasive Reduction and Osteosynthesis System) system, which consists in $4 \mathrm{k}$-wires, 2 introduced from the proximal humeral metafisis and 2 from the fragments of the humeral head, and then threatened by a single external device. Post-operatively, $30^{\circ}$ of 
abduction and flexion were allowed. At a mean follow-up of 13 months (range: 8-24), patients were clinically evaluated with the Constant Score and a radiograph of the shoulder in two-view was obtained.

Results At the follow-up, Constant scores were 58.8 and 18 points for the injured shoulder and 68.9 and 9 points for the opposite shoulder. Reduction and healing of the fracture was good in 22 cases $(84.6 \%)$; in 4 cases, resorption of the greater tuberosity was observed.

Discussion Open reduction and internal fixation is usually recommended in displaced 2- and 3-part proximal humeral fractures, whereas hemiarthroplasty is commonly accepted for the management of 4-part fractures and complex fracture-dislocations. In our series of patients, open reduction was not possible and only conservative treatment or percutaneous pinning was allowed. With this technique, we obtained good results with very low morbidity. The MIROS system should be considered as a valid treatment in these challenging cases.

\section{HEREDITARY OSTEOCHONDRODYSPLASIAS AND NEUROMUSCULAR DISORDERS}

\section{The pelvis and the lower limbs in neuromuscular disorders of the paediatric age: diagnosis and treatment}

\author{
R. Schiavon, A. Micaglio, M. Veronese, G. Turi
}

Orthopaedic and Traumatology Department, San Bortolo General Hospital (Vicenza, ITALY)

Neuromuscular disorders (NMD) recognize genetic, hereditary and degenerative origin. NMD can be associated with chromosome alterations or syndromic diseases and are based on central nervous system affections (e.g., cerebral palsy)/malformations (e.g. spina bifida) or peripheral affections (e.g., hereditary motor and sensory neuropathy); the involvement of muscles is a consequence but a lot of primary muscular disorders are described (e.g., dystrophic and myotonic affections).

NMD can be present at birth or may develop in the first decade of life. Acquired NMD recognizes traumatic, infective, neoplastic, post-surgery, ischemic and post-vaccination etiology.

Clinical manifestations, evolution and prognosis are typical of each type or subtype of condition. At the onset weakness and tonus alterations appear; the progression is characterized by soft tissue contractions, progressive range of motion restrictions, joint subluxations/dislocations, articular and bone deformities. Motor, gait and standing disorders appear at the beginning and the orthopaedist may be requested as a prime consultant when the diagnosis is still unknown. Postural disorders are the common last manifestations. It is important to well establish the internal relations of the dysfunction/deformity of the lower leg as complete and between the pelvis and the body, and the pelvis and the hip.

Subluxation or dislocation of the hip can be often asymptomatic; normally the hip is treated when it correlates with pelvic obliquity, but a severe scoliosis is the main responsible of the pelvis malalignment.

Mio-tenotomies not always are sufficient to correct joint restrictions due to the adhesions of the peri-articular tissues.

Immobilization should be avoided as much as possible especially in disorders associated with weakness.

Common surgical orthopaedic procedures can be performed to release contractures or to prevent/treat joint deformities/subluxations/dislocations. However, surgery is not always necessary and often it will not be successful. In case of operative treatment the goal is restoration of function and posture. Anyway the aim of an anatomic (radiographic) restoration of the deformity is not always achieved and sometimes it is faced the necessity of exceeding it (e.g., performing a proximal valgus luxation osteotomy of the proximal femur) or creating a new deformity (e.g. performing an extension osteotomy of the lower femur in presence of a tight flexion contraction of the knee; performing an astragalectomy, etc.).

We report and discuss a case series composed by a heterogeneous group of 245 patients with NMD who were treated starting from 2000.

\section{Skeletal features of Marfan syndrome}

C. Chillemi ${ }^{1}$, A. Gigante ${ }^{2}$, F. Greco ${ }^{2}$

${ }^{1}$ Istituto Chirurgico Ortopedico Traumatologico (Latina, ITALY);

${ }^{2}$ Clinica Ortopedica, Università di Ancona (Ancona, ITALY)

The Marfan syndrome is a heritable disorder determined by an autosomal dominant gene. It is a disorder of mesenchymal tissues and affects multiple tissues and organs. The Marfan syndrome displays high penetrance variability and an incidence of 2-3/10,000 individuals. The diagnosis is based on the presence of typical clinical features.

The aim of the study was to review the orthopaedic features and describe the management of the most common orthopaedic problems associated with the Marfan syndrome.

Sixteen patients (10 males and 6 females) with a dolichostenomelic habitus and joint hypermobility were included in the study, and after a clinical and radiological evaluation were divided into 3 groups: Group A, 8 patients (marfanoid habitus: skeletal manifestations without extraskeletal involvement); Group $B, 4$ patient (formes frustes: marfanoid habitus + other manifestations) and Group $C, 4$ patients (definite Marfan syndrome).

In all these patients scoliosis was the most frequent musculoskeletal manifestation, but knee and foot deformities also had a considerable incidence. As these features are evident in childhood and adolescence early and accurate orthopaedic evaluation is necessary if progression is to be prevented or halted. A collaborative multidisciplinary approach can help in the diagnosis and prevent the major lifethreatening manifestations typical in this condition.

\section{Melorheostosis: current trends}

\section{Pedrotti, B. Bertani, G. Tuvo, A. Reggiani, R. Mora}

Department of Orthopaedics, Città di Pavia Institute, University of Pavia (Pavia, ITALY)

Melorheostosis is characterized by hyperostotic linear densities in bone, with skeletal aspecific symptoms and abnormalities in skin and vessels. Plain radiographs usually allow for a sure diagnosis. Scintigraphy reveals increased tracer uptake in the bone lesions. CT scan and MRI may accurately localize the demarcation between normal and abnormal bone.

Aetiology was a long-time completely obscure, and the medical treatment was mainly symptomatic. Murray and McCredie [1] suggested a relationship between linear hyperostoses and sclerotomes, and recent researches [2] reported that Melorheostosis is due to a mutation on LEMD3 gene, that encodes for an integral protein of the nuclear membrane. 
Treatment includes conservative methods and surgical methods for soft tissues (capsulotomy, fasciotomy, tendon lengthening) and bone (osteotomy, bone lengthening, arthrodesis, arthroplasty, excision of hyperostotic bone, amputation). Bied et al. [3] proposed the use of Disodium Etidronate (EHDP) administered in high doses to inhibit the formation of hydroxyapatite crystals wit slowing down of bone lesions progression and relief of pain. Other few cases treated with bisphosphonates are described in the literature.

Seven cases of Melorheostosis (2 males and 5 females, with involvement of sclerotomes L5 in 5 patients and S1 in 2 patients) are presently upon treatment at our Department. Excision of hyperostotic bone was necessary in 1 case, where an histological analysis was performed. The patients are treated with administration of EHDP in high doses $(20 \mathrm{mg} / \mathrm{kg} / \mathrm{day})$ for 6 months, with intervals of 6 months to avoid the possible occurrence of osteomalacia, caused by inhibition of mineralization. Periodic X-ray pictures are unchanged, but in every patient a clear improvement of pain and a reduction of limitation of joint motion were observed.

In our opinion the treatment of choice is represented by high dose EHDP, because of its physical- chemical effects (inhibition of calcium phosphate precipitation and of the transformation of inorganic calcium into hydroxyapatite, slowing down of the aggregation of the hydroxyapatite crystals). This treatment can be considered as a valid alternative to the palliative therapy of Melorheostosis.

\section{References}

1. Murray RO, McCredie J (1979) Melorheostosis and the sclerotomes: a radiological correlation. Skeletal Radiol 4:57-71

2. Hellemans J, Preobrazhenska O, Willaert A et al. (2004) Lossof-function mutations in LEMD3 result in osteopoikilosis, Buschke-Ollendorff syndrome and melorheostosis. Nat Genet $36: 1213-1218$

3. Bied JC, Malsh C, Meunier P (1976) La mélorhéostose chez l'adulte. A propos de 2 cas dont l'un traité par un diphosphonate. Rev Rhum Mal Osteoartic 43:193-199

\section{Mesenchymal stromal cells and platelet gel for bone tissue regeneration in congenital pseudarthrosis of the tibia}

\author{
D. Granchi ${ }^{1}$, V. Devescovi ${ }^{1}$, S.R. Baglio ${ }^{1}$, O. Donzelli ${ }^{2}$, \\ M. Magnani $^{2}$, A. Giunti ${ }^{1,3}$, N. Baldini ${ }^{1,3}$ \\ ${ }^{1}$ Laboratory for Orthopaedic Pathophysiology and Regenerative \\ Medicine; \\ ${ }^{2}$ Department of Paediatric Orthopaedics, Rizzoli Orthopaedic \\ Institute (Bologna, ITALY); \\ ${ }^{3}$ Human Anatomy and Pathophysiology of the Locomotor Apparatus, \\ University of Bologna (Bologna, ITALY)
}

Objective Congenital pseudarthrosis of the tibia (CPT) is associated with Type 1 Neurofibromatosis, (NF1) in $50 \%$ of cases, and it is characterized by spontaneous fractures that do not heal. Since the repeated surgical failure often leads to amputation of the limb, it is reasonable to assume that therapeutic strategies based on autologous mesenchymal stem cells (MSC) transplantation, in combination with platelet gel $(\mathrm{PlG})$, can promote bone tissue repair. However, the osteogenic potential of MSC, as well as the microenvironment in which the osteoprogenitors will be transplanted, may be altered on the basis of genetic (NF1+) or congenital defects (NF1). The aim of this study was to evaluate the osteogenic activity of MSC in patients with CPT, and to initiate a clinical trial to evaluate the effectiveness of treatment.
Material and methods MSC were obtained from bone marrow samples collected from tibia, near to the pseudarthrosis site (P-MSC) and from iliac crest (IC-MSC) of 7 patients CPT_NF1+, 6 CPT_NF1and 4 control subjects. Peripheral venous blood were collected from each patient to obtain the autologous serum (AUT) used in the cell cultures. The osteoblast differentiation was induced under standard conditions or in presence of AUT serum, in order to mimic the in vivo using of PlG. Bone fragments collected near to the surgical site allowed to obtain mature osteoblasts, whose supernatant (OB-CM) was used in co-cultures with IC-MSC.

Results Taken together, biochemical, functional and molecular results showed that IC-MSC of children affected by CPT were more osteogenic than P-MSC, but their regenerative potential was lower in comparison to the control group. The defect was more pronounced in $\mathrm{NF} 1+$ patients, probably due to the decrease in neurofibromin function, but in some cases the osteogenic differentiation of MSC was restored by AUT serum. On the contrary, AUT serum inhibited the mineralization process and the expression of some bone-related genes in CPT_NF1 - group. The differentiation of IC-MSC was not affected by OB-CM. Ten patients were treated with MSC and PIG. An effective bone regeneration was obtained in 3/6 patients with CPT_NF1+. Serum levels of FGF2 and the ability of MSCs to mineralize in vitro seemed to be associated with the successful of the treatment.

Conclusions In conclusion, cell therapy could be a useful tool for the treatment of recurrent CPT, because it increases the opportunity to achieve bone consolidation. Laboratory testing performed before surgery could be predictive on the effectiveness of treatment.

\section{Current therapeutic guidelines in Duchenne muscular dystrophy (DMD) to prolong life} V. Riccio, I. Riccio, F. Gimigliano, D. Riccardi, G. Riccardi Duport,
G.Y. Rideau

Dipartimento di Scienze Ortopediche, Traumatologiche, Riabilitative e Plastico Ricostruttive, Seconda Università di Napoli (Naples, ITALY)

Introduction Duchenne myopathy is an autosomal sex-linked disease with an inexorable evolution to death within 20 years of age. This disease presents well defined evolutionary phases, with degradation of the walking function beginning at the age of 3 years and its loss approximately at nine. Immobilization on the wheelchair causes the development of an evolutive scoliosis. At the same time, a progressive degradation of the respiratory function begins and leads the patient to death at a young age. In 1985 Y. Rideau in France carried out a new global therapeutic strategy for the treatment of lower limb deformities, scoliosis deformity and progressive restrictive syndrome. Material and methods In the lower limbs the indication for surgery is made precociously, at the appearance of the first signs of the disease: muscular retractions, with still conserved muscular strength and a almost normal deambulation. The procedures are carried out at the same time and always bilaterally and includes:

- hip: section of superficial flexors

- thigh: iliotibial band resection,

- knee: subcutaneous tenotomy of semitendineous and gracilis;

- foot: subcutaneous lengthening of Achilles tendon, or with a little incision at muscle-tendon junction.

Post-operatively, the patient begins exercises of active and passive mobility and after three weeks has recovered his standards with a deambulation that will remain almost normal for several years. Surgical treatment of Duchenne scoliosis must not be performed too late, 
when the respiratory restrictive syndrome makes the patient inoperable. So, the treatment of scoliosis must be precocious and by using suitable instruments. The indication for early surgery is essentially based on clinical findings and on radiographic balance. Over 10 years ago, in Poitiers, a specific instrumentation for Duchenne scoliosis was designed, providing cylindrical rods fixed by peduncular screws at sacro-lumbar level. On the dorso-lumbar level, the cylindrical rod becomes flat and rectangular, in order to allow more flexibility and to permit little movements of the trunk. Also, the closing wires are under transverse apophyses, not under laminas.

Results The comparison between two groups of patients, the first one precociously operated, the second one not operated, shows that surgery increases the time of normal life. Among the 55 patients operated for scoliosis, we observed rods breaking in two cases and superficial infection in one case.

Discussion and conclusions The goal of lower limb early surgery is to offer patients the opportunity to extend the so-called normal life and to avoid the development of oblique pelvis. Goals of early surgery for scoliosis are to avoid the evolution of scoliosis and to limit the harmful effects of scoliosis on the respiratory function. But surgery alone is not enough to reach the final goal of life extension in these patients. Treating the restrictive respiratory syndrome at first with nasal ventilation and then with tracheotomy is still essential to the survival of the patient.

\section{ORAL COMMUNICATIONS}

\section{KNEE 4}

\section{Patellar non-resurfacing in total knee arthroplasty}

\author{
F. Atzori, A. Massè, C. Cuocolo, A. Nicodemo, C. Sabatino \\ S.C.D.U. Ortopedia e Traumatologia, AOU San Luigi Gonzaga \\ (Orbassano-TO, ITALY)
}

We analyzed retrospectively twenty consecutive cases of patients with primary TKA for osteoarthritis; all the patients had the same type of implant, without patella resurfacing. The study outcomes included the scores according to the Knee Society clinical rating system, the scores according to a patellofemoral-specific patient questionnaire, the radiographic findings, complications and revisions. On the basis of our findings and in agreement with the literature, the indication for arthroplasty of the patella remains controversial, but also in consideration of the type of implants used in our patients it seems useful to take in great consideration this surgery technique, at least in presence of clear indications.

\section{Results of 690 cases of total knee prosthesis implanted using a transepicondilar aligner device between December 2007 and December 2009}

\author{
G. Pipino $^{1}$, D.C. Vaccarisi ${ }^{2}$, G. Maffei ${ }^{1}$ \\ ${ }^{1}$ Ospedale Villa Regina (Bologna, ITALY); \\ ${ }^{2}$ Ospedale S. Maria della Scaletta (Imola, ITALY)
}

The transepicondilar aligner device is a mechanic navigation system that allows rotation alignment of prosthesis femoral component with transepicondilar axis in total knee prosthesis surgery. It is adaptable to every prosthesis instrumentation, easy to use and to understand.

The transepicondilar line is perpendicular to A-P Whiteside line, but while the second is an unclear line, the transepicondilar line is easy to be found since it is close enough to finger epicondyles, that are the more prominent anatomic structures in L-L, to trace the line and to assemble the device.

It is possible to calculate the transepicondilar line on preoperating T.C. and then regulate and adapt the aligner device on the calculated angle.

All 690 TKA were implanted with the same MIS technique, by the same expert surgeon through miniparapatellar approach and three different prosthesis models were used (PS mobile platform, CR mobile platform and CR fixed plate). Results were evaluated by the Oxford Knee Society Score.

The mean rotating angle of transepicondilar line, calculated by the aligner device with posterior Landmark, was of $4.5 \pm 0.5$ of external rotation in valgus knee and $3.5 \pm 0.5 \mathrm{f}$ external rotation in varus knee. This angle was calculated during surgery or on preoperating T.C. Only in 5 cases of varus knee rotation angle of transepicondilar line was 1 degree of internal rotation. They are 5 cases of varus, congenital, intrarotated tibiae.

In this series of 690 TKA, the common constants were femoropatellar pain, no patellar prosthesis, objective or subjective feeling of almost normal joint with ROM between $0^{\circ}$ and $130^{\circ}$.

\section{KNEE 5}

\section{Treatment of recurrent patellar dislocations using a modified Ochi's technique}

\author{
M.A. Natale ${ }^{1}$, A. Caraffa ${ }^{2}$, P. Antinolfi ${ }^{2}$, E. Trinchese ${ }^{2}$, V. Salini ${ }^{1}$, \\ C.A. Orso ${ }^{1}$ \\ ${ }^{1}$ Orthopaedic and Traumatologic Department, \\ University G. d'Annunzio of Chieti (Chieti, ITALY); \\ ${ }^{2}$ Orthopaedic and Traumatologic Department, \\ University of Perugia (Perugia, ITALY)
}

Introduction Recurrent patellar dislocations are a consequence of a patello-femoral instability, which, in turn, derives from a general patellar extensor apparatus disorder. This clinical condition presents itself with recurrent episodes of external patellar subluxations and luxations, even when resulting from mild trauma. It occurs more frequently in females between the years of age 10 and 20, but occasionally also in young male athletes. Treatment of relapsing recurrent patellar dislocations by manifold surgical techniques have already been described.

Material and methods Our case study involved 16 patients ( 9 male and 7 female) with recurrent patellar dislocations treated between January 2008 and September 2009. As surgical treatment we performed a reconstructive operation of the MPFL (medial patellofemoral ligament), following the technique described by Ochi, but replacing the semitendineous tendon with the gracilis muscle tendon. The operation was carried out by distally detaching the tendon and then passing it through a hole drilled in the patella, before being sutured laterally. In this way it was possible to obtain patellar stabilization through the MPFL dynamic reconstruction.

Results All 16 patients were tested periodically during a 6 month follow-up using also biomechanics tests. None of the patients developed any superficial or deep bacterial infections. No ruptures of the reconstructed ligaments were detected. Furthermore, all patients 
completely recovered their knee ROM and, most importantly, no new episodes of dislocation of the operated patella were reported.

Conclusions In conclusion, even with a limited number of individuals, our trial leads us to state that this surgical method, both for its ease in terms of manual surgical technique, short operation time and its high success rate, represents a valid treatment to recurrent patellar dislocations. Our technique led to both short-term and long-term satisfactory results, with significant improvement of articular stability and ROM, and good recovery of both general daily and sport-related activities.

\section{Suggested readings}

1. Crosby E, Insall J (1976) Recurrent dislocations of the patella. J Bone Joint Surg 58A:9-13

2. Christiansen SE, Jacobsen BW, Lund B, Lind M (2008) Reconstruction of the Medial Patellofemoral Ligament With Gracilis Tendon Autograft in Transverse Patellar Drill Holes. Arthroscopy 1:82-87

3. Fernandez E, Sala D, Castejon M (2005) Reconstruction of the medial patellofemoral ligament for patellar instability using a semitendinosus autograft. Acta Orthop Belg 71:303-308

4. Deie M, Ochi M, Sumen Y, Adachi N, Kobayashi K, Yasumoto M (2005) A long-term follow-up study after medial patellofemoral ligament reconstruction using the transferred semitendinosus tendon for patellar dislocation. Knee Surg Sports Traumatol Arthrose 13:522-528

5. Aglietti P, Buzzi R, De Biase P et al. (1994) Surgical treatment of recurrent dislocation of the patella. Clin Orthop Rel Res 308:8-17

6. Lim AHS, Chong Chang H, Hui JHP (2008) Recurrent Patellar Dislocation: Reappraising our Approach to Surgery. Ann Acad Med Singapore 37:320-323

\section{Preliminary validation of a non-invasive device used to quantitatively assess Pivot-Shift test}

\author{
S. Zaffagnini ${ }^{1}$, C. Signorelli ${ }^{1,2}$, N. Lopomo ${ }^{1}$, S. Bignozzi ${ }^{1}$, A. Visani ${ }^{1}$, \\ M. Marcacci ${ }^{1}$ \\ ${ }^{1}$ Istituto Ortopedico Rizzoli (Bologna, ITALY); \\ ${ }^{2}$ Politecnico di Milano (Milan, ITALY)
}

Introduction During the last years several devices have been developed in order to quantify the level of ACL injuries; moreover Pivot-Shift (PS) test is becoming commonly used to assess knee dynamic instability, that is correlated with ACL injury. The objective of our study was to clinically validate a non-invasive system able to quantify PS-test; a set of clinical parameters able to characterise PS-test and to achieve a quantitative analysis of ACL pathology was therefore identified.

Patients and methods PS test was repeated on 35 consecutive ACL patients by an expert and a novice surgeon on pathologic and healthy limb. Experimental set-up consisted of a sensor that allowed to acquire acceleration signal reached during PS test. This device was skin-fixed on the tibia between the lateral aspect of anterior tuberosity and Gerdy's tubercle. The identified set of parameters included: maximum (MAX) and minimum ( $\mathrm{min}$ ) value of the acceleration reached during the manoeuvre, difference (Diff) and an index of the smoothness of the test (in physics indicated as Jerk). ICC analysis was used to assess intra and inter-tester repeatability, while Student $t$-test was used to explore the statistical differences between limbs (healthy and injured) and conditions (pre- and post-anaesthesia) with $p=0.05$.
Results The obtained results showed a good reliability, with a mean Inter-tester ICC of $0.61(p<0.05)$. Pathologic limbs, compared to the healthy limb, revealed a lower min value of acceleration $(8.138 \mathrm{vs}$. $\left.8.539 \mathrm{~m} / \mathrm{s}^{2} ; \quad p=0.0003\right)$, a higher jerk $\left(31.73 v s .23 .51 \mathrm{~m} / \mathrm{s}^{3}\right.$; $p=0.0407)$ and a higher diff (3.807 vs. $\left.2.682 \mathrm{~m} / \mathrm{s}^{2} ; p=0.0019\right)$. Anaesthesia did not introduce any significant difference in PS outcome (condition comparisons had a $p \gg 0.05$ ).

Conclusions We aimed to a non-invasive and reliable method that allowed a quantitative evaluation of PS test usable both in clinical practice and operating room. The statistical description of PS outcomes reported a significant difference between the injured and the control-lateral limb: the identified parameters result representative of the ACL pathological condition.

\section{Posterolateral corner reconstruction using a modified LaPrade technique}

G.F. Trinchese, G. Iervolino, G. Calabrò, L. Prinzo, F. Tripodi, A. Toro

Department of Orthopaedic Surgery, G. Fucito Hospital, (Mercato San Severino-SA, ITALY)

Objective Injuries to the posterolateral corner (PLC) of the knee produce a spectrum of pathologic states of ligamentous laxity resulting in various amounts of varus, external rotation, and posterior translation of the knee. Consequently, they may result in significant functional disability. For years, posterolateral corner injuries have been a challenging diagnostic and therapeutic problem for the orthopaedic surgeon. Various surgical techniques to treat posterolateral knee instability have been described. The goal of this study is to determine the clinical outcome of PLC reconstruction following the LaPrade technique modified by the authors.

Material and methods We retrospectively studied 5 patients who underwent a PLC reconstruction with the LaPrade technique modified by the authors, from September 2007 to November 2009. Three patient had a concomitant ACL rupture, one an isolated PLC injury and one a residual posterolateral instability after a previous ACL reconstruction. In the modified technique the reconstruction of PLC was performed with a single femoral tunnel, using tibialis anterior allograft for the popliteus-popliteofibular reconstruction and peroneus longus allograft for the collateral ligament reconstruction. In the cases of concomitant ACL lesion the reconstruction was performed using a tibialis posterior allograft. Mean age was 30.2 years (range 16-40). Mean follow-up was 21.4 months (range 9-35). All patients underwent the same postoperative protocol.

Results We evaluated varus stress test, recurvatum test, dial test, manual Lachman and pivot shift tests, maximum anterior tibial translation by Rolimeter, Lysholm and IKDC forms. All patients showed negative dial and recurvatum tests; one showed $1+$ on varus stress. Mean Lysholm score was 93 points (range 82-100). On IKDC evaluation 1 patient was grade $\mathrm{A}$ and four were grade $\mathrm{B}$.

Conclusions Although clear limits of the study due to small number of cases, the modified LaPrade technique showed good results in terms of clinical outcome, restoring varus and rotation stability of knees in treatment of chronic PLC injury.

\section{KNEE 6}

No Abstracts were received for this Section. 


\section{MISCELLANY}

\section{Muscle involvement in rheumatoid foot: histological and ultrastructural findings}

\author{
M. Marinelli, L. Memè, M. Pavan, L. de Palma
}

Cattedra di Ortopedia e Traumatologia, Università Politecnica delle Marche, Azienda Ospedaliero-Universitaria Ospedali Riuniti (Ancona, ITALY)

Rheumatoid Arthritis (RA) is a chronic inflammatory disease characterized by the involvement of numerous internal organs and tissues although the major target are joints. Patients with RA often show stiffness and reduced muscular function that are generally ascribed to the clinical conditions of the patients but that might be due to the muscular involvement of the disease itself.

The existence of rheumatoid myositis is doubtful and few data in literature show the typical ultrastructural characteristics of rheumatoid myositis.

The aim of the present study is the description of the histological and ultrastructural aspects of muscles in the attempt to find out typical characteristics of RA inflammation.

We studied two groups of patients: (1) Group A: 12 patients ( 8 female, 4 male, age: $36-72$ years) affected by AR, with a disease duration of 6 months -42 years; therapy: NSAID (24 patients), golden salts (10 patients), steroidal therapy (6 patients); (2) Group B: 12 healthy patients sex and age matched.

Biopsies were taken from the "extensor digitorum" of the foot and analysed with optical microscopy and electronic microscopy. Specimens were fixed, paraffin embedded and treated with ematossilineosin or Mallory method. Specimens for electronic microscopy were fixed in cacodylate, treated with osmium tetroxide and uranil acetate. After alcohol dehydration they were immersed in epoxy resin.

Optical Microscopy The peculiar aspects are: chronic inflammatory infiltration, reduced number of muscular fibers with variable diameter, mononuclear cells infiltration in skeletal muscle.

Electronic Microscopy The peculiar features are: mononuclear cells infiltration into vessels wall, myofibers separation, presence of glycogen particles between the myofibers, mithocondrial polymorphism and sarcotubular system dilatation, myelinic figures, presence of lipofuscin particles under the cellular wall.

This study show the existence of a muscular damage due to the rheumatoid disease itself (rheumatoid myositis) and independent on other causes (vasculitis, neurophaty, etc.).

Rheumatoid myositis is characterised by some typical histological and ultrastructural aspects that could be useful in the characterization of the disease.

\section{Medico-legal aspects in the orthopaedic use of "platelet-rich plasma"}

\author{
G. Martini ${ }^{1}$, D. Palmieri ${ }^{2}$, L. Ottaviano ${ }^{2}$, M. Mastrangelo ${ }^{3}$ \\ ${ }^{1}$ Commissione di Medicina Legale SIOT (Rome, ITALY); \\ ${ }^{2}$ Gruppo di Studio Commissione di Medicina Legale SIOT \\ (Rome, ITALY); \\ ${ }^{3}$ U.O. Ortopedia e Traumatologia, Zona Territoriale 9 Macerata \\ (Macerata, ITALY)
}

Introduction The relationship between technological innovation, social changes and legal solutions always poses new problematic towards how often appear completely unsuitable the old criteria of appraisal.

Objective We wish to analyze some possible causes of responsibility in the use of platelet-rich plasma: the increasing use of platelet-rich plasma (PRP) in orthopaedics presents significant opportunities as well as significant questions about medico-legal issues as regarding in particular the indications, the use of guidelines (Leges Artis), the informed consent and also the control/authorization of Transfusion Centers.

Discussion and conclusions Innovative choices involve not always the well known problem concerning the not sufficiently acquired skills, but also the prudence is not motivated entirely and the diligence, only generic, is not detailed to solve problems of unknown impact. If the generic operating risk is known in its general aspects, the specific ones, in an innovative surgery, can be entirely not predictable also in terms of biological reactions. As concerning innovative choices, also the Magistracy expresses open judgments inspired more to a rigorous verification that to a trust that should be granted to those, who crossing a new road, showed to have found a path leading to notable clinical advantages. Anyway with the evolution of technological innovation as the use of platelet-rich plasma (PRP) in orthopaedic practice, it cannot be neglected the necessity of adopting more suitable rules to manage both scientific and social truth with the aim of linking the progress of the scientific acquisitions with the legal thought as well as the law development.

\section{Retrospective study of the use of paracetamolo/ tramadolo association for pain treatment in osteoarthritis}

\author{
D. Brioschi, R. Franceschini
}

UO Ortopedia e Traumatologia, Azienda Ospedaliera L. Sacco (Milan, ITALY)

Introduction The recent increase of the restrictions in the use of the traditional anti-inflammatory drugs and COx-Inhibitor, associated to extensive use of other drugs in the elderly population, has pushed us to study the clinical effect of the paracetamolo/tramadolo association in the treatment of the secondary pain to arthrosis. In these patients it is necessary to consider the frequent concomitant therapies and the problematic pharmacokinetics for accumulating phenomena [1-3].

Material and methods In this retrospective study 24 patients affected by osteoarthritis were enlisted (age comprised between 46 and 94 years). $37.5 \%$ of the subjects had lumbar arthrosis, $66.7 \%$ knee arthrosis, $12.5 \%$ hip arthrosis, $12.5 \%$ thumb arthrosis, $12.5 \%$ cervical arthrosis and $16.7 \%$ cervical arthrosis. Before the study, $83.3 \%$ of the patients had been treated with analgesic pharmacologic treatment (NSAID, paracetamol, opioids). Such treatments turned out little effective in $70.8 \%$ and ineffective in $25 \%$ of the cases. All the patients were dealt with paracetamolo $(325 \mathrm{mg}) /$ tramadolo $(37.5 \mathrm{mg})$ association (Kolibri) for a period comprised between 12 and 67 days. The effectiveness of the treatment was evaluated by estimating the intensity of pain at rest and on activity with a $0-3$ points scale, the quality of the sleep (a lot disturbed, frequent awakenings, bond, deep), the daily autonomy and the reduction of the working activity. The variables were analyzed by Wilcoxon method for joined data.

Results The paracetamolo/tramadolo association reduced pain both at reast and on activity in a statistically significant measure $(p<0.01)$; No pain of serious degree was recorded at the final visit. The quality of sleep was also improved in a significant way $(p<0.01)$. The daily autonomy evidenced a statistically significant improvement. The treatment turned out very effective in $20.8 \%$ of the patients, effective 
in $41.7 \%$ and ineffective in 2 patients $(8.3 \%)$. The collaterals effects were modest.

Conclusions The data obtained from this study demonstrated the good analgesic effectiveness of the paracetamolo/tramadolo association associated with an optimal tolerability in the treatment of osteoarthritis. Such treatment is to be considered valid and lacking of important toxicity also in elderly patients.

\section{References}

1. Klotz U (2003) Tramadol - the impact of its pharmacokinetic and pharmacodynamic properties on the clinical management of pain. Arzneim Forsch Drugs Res 53(10):681-687

2. Grond S et al. (2004) Clinical Pharmacology of tramadol. Clin Pharmacokinet 43(13):879-923

3. Cossmann M et al. (1995) General tolerability and adverse event profile of tramadol hydrochloride. Rev Contemp Pharmacother 6:513-531

\section{HIP 6}

\section{The role of obesity and the choice of implant in hip arthroplasty}

F.R. Evola, V. Pavone, L. Costarella, G. Caff, G. Sessa

Orthopaedic Clinic, University of Catania (Catania, ITALY)

Objective Multiple studies showed an increase of general complications, like pulmonary embolism or heart failure, operative time, blood loss, post operative fever, hospital days, and the reduction of functional score in obese patient after total hip replacement (THA) [1]. Aim of the present study is to evaluate the mid term results of the incidence of orthopaedic complications in obese and non obese patents after THA performed at the Orthopaedic and Traumatologic Clinic of the University of Catania.

Material and methods Between January 2000 and December 2009, 895 THA were performed in 565 non obese patients and 330 obese patients; the mean age was 67 years (range $41-81$ years).The group of study comprised 402 male and 493 female, operated for degenerative disease in 558 cases and for traumas in 337 cases. The implants were followed both clinically, using the Merlè-D'Aubigne-Postel scale ( $0-18$ points), and through the conventional radiology with serial control at 3, 6 and 12 months and every year.

Results The average follow-up was 7 years (range 1-11 years). Clinical results were good with a mean score that increased from 6 to 15 points in normal patients and from 4 to 11 in obese. We did not find a significant relationship between obesity and the incidence of medical and orthopaedic complications. In our study, no statistically significant difference was found on early failure between cemented and cementless implants in obese patients. The survivorship of total hip arthroplasty is similar in obese and non obese patients.

Discussion Adults are classified as overweight or obese (class $1^{\circ}, 2^{\circ}$, or $3^{\circ}$ ) according to the body mass index (BMI). There is increasing evidence that obese patients are more likely to require THA than non obese patients. The high preoperative BMI is associated with decreased ambulation; the detrimental effects of high BMI on physical function are not directly linked to the biomechanical functionality of the hip joint itself, but to the overall agility and mobility of patients.

Conclusions Although obese patients may have increased loads through their hip arthroplasty, there is evidence that the increased weight is balanced by decreased cycles of loading. These data suggest that body weight should not be a justification for withholding surgery from overweight or obese patients, and a long term follow-up is required to evaluate implant longevity.

\section{Reference}

1. Yeung E, Jackson M, Sexton S, Walter W, Zicat B, Walter W (2010) The effect of obesity on the outcome of hip and knee arthroplasty. Int Orthop [Epub ahead of print 29 May 2010]: DOI 10.1007/s00264-010-1051-3

\section{Modular porous tantalum implants in prosthetic reconstructive surgery of the hip}

N. Fabbri, P. Ruggieri, F. Vommaro, E. Rustemi, A. Angelini, M. Mercuri

Istituto Ortopedico Rizzoli (Bologna, ITALY)

Objective Durable acetabular fixation may be difficult to achieve when significant bone defect and/or poor quality of residual bone stock are present. Purpose of this study was to review clinical results of patients with severe acetabular bone loss managed with modular porous tantalum implants.

Material and methods Retrospective study of 16 patients that underwent revision THA (13 patients, 14 implants) or primary THA (3 patients, 3 implants) in the setting of significant acetabular bone defect. There were 10 females and 6 males, for a total of 17 THA procedures ( 1 case of bilateral revision surgery); mean age 53.5 years (26-77 years). All the patients had a mixed cavitary and segmental acetabular bone defect, which according to Paprosky was classified as follows: $2 \mathrm{~A} / 2 \mathrm{~B}$ : 2 cases; $2 \mathrm{C}$ : 2 cases; $3 \mathrm{~A} / 3 \mathrm{~B} / \mathrm{massive}$ acetabular: 13 cases. In 4 cases, the defect was associated with pelvic discontinuity. Bone defect was managed in all patients with modular porous tantalum implants, uncemented at the interface with host bone and cemented in the contact zone with augments, associated to the cup in 13 cases, and with morcellised bone grafts to fill the residual cavitary defects and the interstices around implants. Fixation was implemented in 1 case by a "cup-cage" type of construct in 1 case. The minimum follow-up was 2 years in all cases, mean follow-up was 3.5 years (2-6.5 years).

Results There were no re-operations. There was 1 early dislocation successfully managed conservatively. In all cases the acetabular implant was well fixed and functional at the most recent follow-up.

Conclusions Porous tantalum has shown very satisfactory results at short term follow-up, demonstrating reliable capability to obtain biologic fixation in THA revision surgery and becoming our treatment of choice for patients with severe acetabular bone loss. Longer follow-up is required to appreciate long-term shortcomings.

\section{HIP 7}

\section{Preliminary results on joint function in patients} with ceramic-ceramic coupling of large diameter

\section{Lucidi, C. Barresi, M. Manili, R. Giacomi}

\section{San Carlo Hospital (Rome, ITALY)}

Objective Favorable results in terms of wear, quantity and toxicity of debris derived from the introduction of hip arthroplasty with ceramicpolyethylene, ceramic-ceramic and metal-metal coupling at the 
junction of head/cup are well known and documented. If, at present, the ceramic-polyethylene coupling is the golden standard in hip arthroplasty, also the ceramic-ceramic coupling, with decennial application, is a clinical reality of great significance and value. Ceramic-ceramic couplings have the lowest wear rates and minimal production of debris. These results are nearly devoid of toxicity. The phenomena of rupture of the components (heads or acetabular inserts), so frequent in the past, have now become exceptional. Besides, the use of heads with larger diameter than before (36-38 mm) allows a greater range of motion and significantly reduces the risk of implant dislocation. It follows that systems with ceramic-ceramic coupling and large diameter heads are the tribological choice option in young active patients.

Material and methods We report a review of literature on this subject, the rationale and experience in the use of arthroplasty with ceramic-ceramic coupling and heads of large diameter.

Conclusions The use of arthroplasty with large heads and ceramicceramic coupling, is certainly capable of ensuring a greater range of motion and less friction, thus reducing the risks of dislocation and wear and significantly improving the survival of arthroplasty.

\section{Our experience with ceramic in arthroplasty}

\section{F. Benazzo, S.M.P. Rossi, C. Russo, L. Piovani}

Clinica Ortopedica e Traumatologica, Università degli Studi di Pavia, Fondazione IRCCS Policlinico San Matteo (Pavia, ITALY)

The use of ceramic in prosthetic surgery dates more than 30 years with first use in total hip replacement. The evolution of this material, that was pure Alumina at the beginning (Aluminium Oxide Al2O3), have been very important with the introduction in the ' $90 \mathrm{~s}$ of the ceramics BIOLOX, BIOLOX Forte and than BIOLOX Delta which allowed, thank to better guarantees concerning the risk of fracture and fragility, to increase the use in hip replacement and the introduction in knee replacement procedures, field where the experience was only from the Japanese community

Ceramic is anallergic, can be used in atopic patients and patients allergic to metal ions, it has very good tribological characteristics, either in the cer-cer coupling, or coupled with PE, where, in the knee especially, it exists an advantage comparing to $\mathrm{CrCbMo}$ alloy concerning the wear issues

We performed, from 1999 to March 2009, 511 implants in 465 patients (46 bilateral). 320 patients were women, 145 men. Mean follow-up is 5.3 years ( 6 months-10 years). Mean age was 68.4 years (18-80).

The mean Harris Hip Score was 93.2 considering the overall population at last follow-up (mean 5.3 years, range 6 months-10 years). In 1 case we experienced the fracture of the liner. 5 implants had dislocations and 1 case needed revision. The other 4 implants were treated conservatively with excellent results at final follow-up.

We had 5 cases of squeaking: in 1 case it recovered itself, in 2 cases the phenomenon was occasional and in 1 case it was persistent. No revision surgery has been required by these 4 patients. 1 patient developed squeaking after a subdislocation and needed revision for substitution of the head.

We revised 3 other implants: 2 for infection and 1 for a periprosthetic fracture.

In knee surgery we followed perspectively 10 Multigen Plus Implants in ceramic implanted in 10 patients between February 2007 and January 2008. At a mean 2.4 years follow-up HSS was 89.6, and KSS was 89.2 concerning the "knee score" and 88.6 concerning the "functional score".

\section{The use of tantalum acetabular components of in total hip replacement}

\author{
F.R. Evola, L. Costarella, V. Pavone, A. Graceffa, G. Sessa
}

Orthopaedic Clinic, University of Catania (Catania, ITALY)

Objective The porous tantalum is a material alternative to those commonly used in primary hip replacement and revision, such as stainless steel, cobalt-chromium alloy and titanium [1]. This metal has a high volumetric porosity, low flexibility modulus, high coefficient of friction that may promote a better biological fixation of implants to bone.

The aim of this study was to evaluate at short-term follow-up the results of prosthetic implants implanted at the Orthopaedic Clinic of Catania using tantalum acetabular component.

Material and methods From October 2006 to December 2009, 32 hip prostheses using tantalum were implanted: 26 cup revisions and 6 primary arthroplasties. The group of study comprised 14 male and 18 female; the mean age was 45 years in primary implants and 73 years in revisions. The implants were followed both clinically, using the MerlèD'Aubigne-Postel scale (0-18 points), and through the conventional radiology with serial control at 3,6 and 12 months and every year.

Results The average follow-up was 21 months for primary implants and 19 month for revisions. The clinical and radiographic examination performed at regular intervals have shown satisfactory results in both primary implants and revisions. Clinical results were good with a mean score that increases from 7 to 15 points in primary implants and from 4 to 12 in revisions. Radiographically there were no signs of mobilization or migration of implants, liner wear, penetration of the femoral head, mobilization of inserted augments.

Discussion The severe reductions of bone-stock represents a major obstacle during a revision surgery of hip prosthesis, especially in the presence of segmental or cavitary bone defects. The tantalum cups represent a valid alternative to traditional acetabular components, even where contact with the host bone is less than $50 \%$, showing to be a reliable and safe material [2].

Conclusions The Trabecular Metal showed a higher mechanical stability and bone integration greater than conventional metals. Despite the considerable cost and the excellent results achieved by this material, both clinical and radiographic studies are needed with larger series and longer follow-up.

\section{References}

1. Balla VK, Bodhak S, Bose S, Bandyopadhyay A (2010) Porous tantalum structures for bone implants: fabrication, mechanical and in vitro biological properties. Acta Biomater 6(8):3349-3359

2. Simon JP, Bellemans J (2009) Clinical and radiological evaluation of modular trabecular metal acetabular cups. Short-term results in 64 hips. Acta Orthop Belg 75(5):623-630

\section{Use of 3D models and virtual procedure simulation in difficult cases of hip replacement surgery}

\author{
M. Lisanti ${ }^{1}$, P. Parchi ${ }^{1,2}$, E. Bonicoli ${ }^{1}$, L. Andreani ${ }^{1}$, V. Ferrari ${ }^{2}$, \\ M. Carbone ${ }^{2}$, M. Ferrari ${ }^{2}$, F. Mosca ${ }^{2}$ \\ ${ }^{1} 1$ st Orthopaedic Clinic, ${ }^{2}$ EndoCAS Center, University of Pisa \\ (Pisa, ITALY)
}

Introduction In the last years new surgical procedures have been developed to improve the quality of treatment, reduce the patients risk 

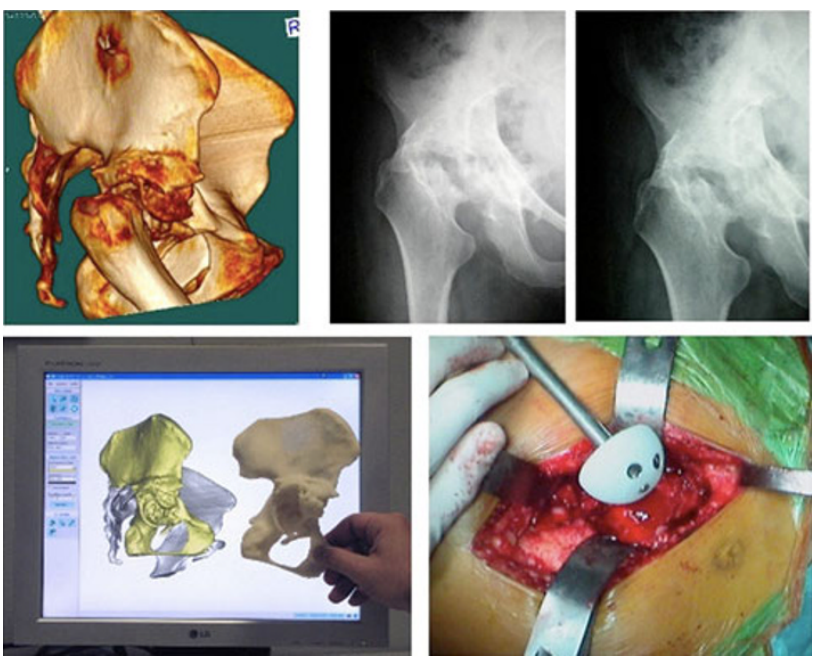

Fig. 1

and improve the clinical outcome. We propose an alternative to intraoperative navigation to approach difficult case of hip replacement based on 3D virtual models, generated by segmenting patients CT images, 3D solid models, obtained by rapid prototyping, and virtual procedure simulation.

Material and methods From March 2009 we have treated 8 patients with a high grade hip destruction due to the sequelae of hip dysplasia, childhood hip disorders and trauma. Patient specific 3D models are generated using a semi-automatic tool developed by us. 3D models obtained segmenting radiological images, allow us to plan and simulate interventions. The 3D virtual model is also used to generated a 3D 1:1 solid model using a 3D printer in order to allow the surgeon to directly understand the bone morphology and test the implant placement before the real surgical procedure.

Results The main advantages of this type of preoperative planning are the direct visualization of acetabular morphology, an accurate choice of the implant (type, size, shape) and an accurate study of the optimal implant position before surgery. In the following we show a paradigmatic clinical case of complex hip reconstruction in a 23-year-old woman who sustained a shotgun lesion at the right hip (Fig. 1). For the complexity of the acetabular morphology the segmentation data are used to make a 3D solid 1:1 model that the surgeon directly used to plan the surgical procedure.

Conclusions Planning the intervention using a segmented dataset allow surgeon to afford critical interventions with a major awareness of the patient specific anatomy and in some cases to choose more efficient surgical approaches. The use of 3D solid model allows the surgeon to know, the exact bone morphology and the implant characteristics with great advantages in terms of reduction of the surgical time and of accuracy of the reconstruction.

\section{SPINE 1}

\section{Surgical treatment of degenerative lumbar scoliosis}

C. Faldini, S. Pagkrati, M. Nanni, D. Leonetti, M.T. Miscione, F. Acri, S. Giannini

Istituto Ortopedico Rizzoli (Bologna, ITALY)
Objective Degenerative lumbar scoliosis occurs in the aging spine without previous history of scoliosis and consists of asymmetric narrowing of the intervertebral space producing lateral spine deviation and segmental stenosis. Degenerative lumbar scoliosis can lead to severe back pain and progressive radiculopathy due to segmental spinal instability and central or foraminal stenosis. The aim of this paper is to present the guidelines for surgical treatment of degenerative lumbar scoliosis based on 54 consecutive cases treated.

Material and methods Fifty-four patients affected by degenerative lumbar scoliosis were treated between 1997 and 2006. DLS involved one level in 12 cases, two in 27,3 or more levels in 15 . Surgery was planned according to the following labelling factors: extension of the DLS, instability, presence of central or foraminal stenosis, neurological involvement, pain. Surgical treatment consisted of: minimally invasive hemilaminectomy and foraminotomy in 5 cases, posterior stabilization and decompression in 14 cases, posterior stabilization and intervertebral height restoration by PLIF in 35 cases.

Results Preoperative curve was $22^{\circ}+5$, at follow-up was $7^{\circ}+5$ $(p<0.05)$. Preoperative VAS pain was $7.5+3$, at follow-up was $2.9+2(p<0.05)$. At the last available follow-up of $4( \pm 2.5)$ years, 29 cases had excellent results, 16 good, 4 fair and 2 poor. One patient treated by foraminotomy needed a second operation of posterior stabilization.

Conclusions Surgical treatment of degenerative lumbar scoliosis has a positive cost/benefit ratio for the patient's condition if the labelling factors of each deformity are carefully studied, to decide the correct surgical option.

\section{Causes of post-surgical instability at the lumbar spine: learning from revision surgery}

\section{N. Papapietro, A. Di Martino, V. Denaro}

Ortopedia e Traumatologia, Università Campus Bio-Medico (Rome, ITALY)

Objective Aim of this paper is to review the intraoperative findings of patients affected by Failed Back Surgery Syndrome (FBSS) and subjected to revision lumbar spine surgery at our Institution in the last 15 years, in order to analyze risk factors for post-operative instability. Material and methods Eighty-nine patients ( 37 female and 52 males, average age 57 years) operated between 1990 and 2005 of revision lumbar spine surgery at our Institution and followed-up for an average of 6 years were included in the study. No patients had had spinal fusion at first surgery. Exclusion criteria were patients operated for recurrent disc herniation and subjected to disc herniation removal, patients with previous instrumented fusion, and patients operated for degeneration adjacent to a previous fusion. Intraoperative findings were recorded. All the patients were subjected to decompressive laminectomy and instrumented fusion via a posterior approach. Oswestry Disability Index was administrated preoperatively, 6 months postoperatively and yearly thereafter.

Results At the first surgery, in 65 out of 89 patients (73\%), arthrectomy (either monolateral or bilateral) plus discectomy was performed. In 17 patients pseudarthrosis in non-instrumented fusion was found. Four patients were subjected to laminectomy alone. Three patients were subjected to discectomy in spondylolisthesis. Intraoperatively, we found in 56 out of 65 artrhectomy patients (86\%), a demolition of more than $50 \%$ of the articular surface. There was significant improvement in the Oswestry Disability Index over all time intervals postoperatively.

Discussion and conclusions In our casuistic, arthrectomies more 50\% associated to discectomy procedures are highly associated to iatrogeneic instability (73\% of our casuistic of revision surgery). If such 
demolition is required to decompress the neural structures, an instrumented fusion should be routinely associated to neural decompression at first surgery.

\section{Revision strategy in cervical multilevel surgery with anterior approach}

\author{
M. Costaglioli, M. Piredda, S. Casula, S. Caboni, P.P. Mura
}

CDC Polispecialistica S. Elena (Cagliari, ITALY)

Objective The study objective is to analyze the role of the anterior approach when revision surgery is required for failures of anterior cervical discectomy and fusion with cage stand alone (ACDF-CA) 3 or more levels.

Background In literature, redo surgeries for failed anterior fusions showed an increased success rate when the approach used is only posterior (94-98\%) and with circumferential fusion (94-100\%). While the anterior only revision shows a $55-57 \%$ success rate. In our experience anterior revision is useful in cases of removal of failed implants, resection of pseudarthrosis, and correction of the anteriorly located deformity. Extending fusion length acquires another intermediate point of fixation and strengthens the overall construct stability thus avoiding additional posterior fixation.

Material and methods A total of 8 consecutive patients with previous surgery for degenerative multilevel instability and spondylotic myelopathy were analyzed. These patients suffered from neck pain or cervical radiculopathy and thus required reoperation. Revision surgery was performed by anterior only approach. Standing plain lateral radiographs and MRI was performed before and after the redo surgery. Fusion rate and subsidence rate were assessed by radiographs. A minimum follow-up of 6 months was considered. Clinical outcomes were assessed using SF-36 evaluation test.

Results In our series there were 5 patients with previous 3-level ACDF-AC and 3 patients with previous 4 levels ACDF-AC. The goal of revision surgery was to correct the size of the cage, pseudarthrosis,

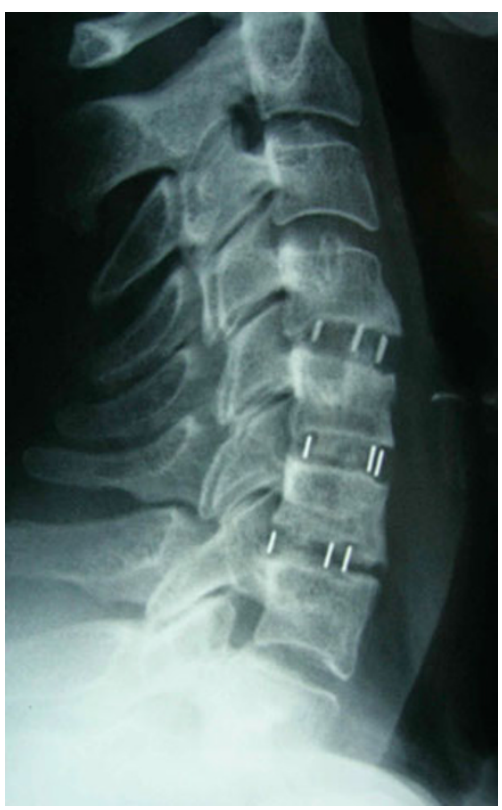

Fig. 1 subsidence and sagittal alignment. We observed significant improvements of clinical outcome related to the correction of these components. Solid fusion was achieved in $90 \%$ of subjects. Subsidence occurred in 2 cases.

Discussion and conclusions In revision surgery for failed ACDF$\mathrm{AC}$ at 3 or more levels, the anterior approach only, with locking plate systems, permits stronger anterior fixation and can avoid the need for posterior supplemental fusion. The use of cage and plate construct in 3 or more-levels results in a more lordotic alignment, an increased disc height, a higher fusion rate, a lower subsidence rate, and a lower complication rate than that of cage alone implants.

\section{The minimally invasive lumbar interbody fusion access through extreme lateral approach: analysis of medium- term results}

A. Zachos $^{1}$, A. Ruggiu ${ }^{1}$, F. Barca ${ }^{1}$, P. Tranquilli-Leali ${ }^{1}$, F. Muresu ${ }^{1}$, M. Balsano ${ }^{2}$, C. Doria ${ }^{1}$

${ }^{1}$ Orthopaedic Department, University of Sassari (Sassari, ITALY);

${ }^{2}$ Orthopaedic Department (Schio, ITALY)

Background The interbody fusion using extreme lateral approach (XLIF), is performed through a percutaneous allowing passage through the retroperitoneal space $[1,2]$. The procedure needs the help of a retractor which is monitored. The exposure allows the direct visualization of the lateral side of the disc, facilitating discectomy and the placement of implants. The surgical results have proven this procedure as a safe and reproducible technique, with the benefits of a minimally invasive procedure, quick recovery and improvements in pain and function scales.

Objective We analysed the clinical and radiographic results of a homogeneous series performed during 2 years in two Italian Centers. Material and methods From January 2008 to January 2010, 22 patients underwent lumbar arthrodesis by XLIF (Fig. 1). In all the cases, the pre-operative diagnosis was degenerative disc disease with radiculopathy. The treated levels were L2-L3 in 7 cases, L3-L4 in 11 cases, and L4-L5 in 4 cases. Evaluation criteria considered the clinical

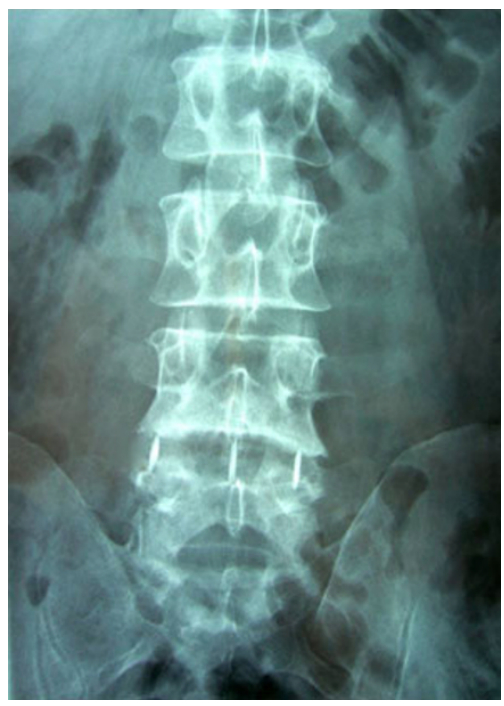

Fig. 1 Lumbar arthrodesis by XLIF 
and radiographic results with the help of pre- and post-operative scales: VAS and ODI.

Results Patients were evaluated at a mean follow-up of 12 months (range 1-24 months). In one case we observed the subsidence of the cage in the lower vertebral body and in one case the cage was placed too back. In about one half of the patients we found a postoperative temporary cruralgia. We observed an improvement on the VAS and ODI scale. The post-operative radiographs made at a distance of 3,6 and 12 months showed an excellent fusion.

Conclusions The lumbar interbody fusion access through extreme lateral side is a minimally invasive technique and also very safe [3]. This technique requires a long learning curve. It's a valid alternative technique preferable to use in elderly patients who could not deal with surgery heavy blood loss.

\section{References}

1. Wright $N$ (2005) Instrumented extreme lateral interbody fusion (XLIF) through a single approach. Proceedings of the NASS 20th Annual Meeting/Spine J 5[Suppl 4]: S177-S178

2. Pimenta L (2001) Lateral endoscopic transposas retroperitoneal approach for lumbar spine surgery. Paper presented at: VIII Brazilian Spine Society Meeting; Belo Horizonte, Minas Gerais, Brazil

3. Ozgur BM, Aryan HE, Pimenta L, Taylor WR (2006) Extreme Lateral Interbody Fusion (XLIF): a novel surgical technique for anterior lumbar interbody fusion. The Spine Journal 6:435-443

\section{Effectiveness of a "global postural reeducation" program for chronic low back pain: a non-randomized controlled trial}

F. Bonetti, S. Curti, S. Mattioli, R. Mugnai, C. Vanti, F.S. Violante, P. Pillastrini

\section{University of Bologna (Bologna, ITALY)}

Objective As regards the treatment of LBP, exercise therapy appears to be slightly effective for decreasing pain and improving function [1]. A review of the literature on GPR suggested that this method may be effective for treating some musculoskeletal diseases and disorders such as ankylosing spondylitis, LBP and lumbar disc herniation [2]. Aim of this non-randomized controlled trial was to evaluate the effectiveness of a Global Postural Re-education (GPR) program as compared to a Standard Physical Therapy Treatment (ST) in subjects with chronic low back pain (LBP) at short- and mid-term follow-up (3 and 6 months).

Patients and methods Two groups of outpatients with a diagnosis of chronic LBP were enrolled in the study: 50 in the GPR group and 50 in the ST group. Basic demographic data (age, gender, BMI), smoking habits, work-related characteristics and educational level as well as duration of symptoms and previous treatment were recorded. Primary outcome measures were Roland and Morris Disability Questionnaire and Oswestry Disability Index. Data were captured at baseline and at $3 / 6$ months.

Results Comparing the differences between groups at short- and midterm follow-up, the GPR group revealed a significant reduction (since baseline) of all the outcome measures with respect to the ST group, using the intention to treat approach to analyze participants according to the group to which they were originally assigned. These results appeared significant both at short- and at mid-time follow-up.

Conclusions In conclusion, this study showed a significant improvement on disability and intensity of pain employing a GPR program, as compared to a conventional physical therapy approach, in patients with chronic LBP. These results must be confirmed by further studies with higher methodological standards, including randomization, larger sample size, of the LBP subjects. The effectiveness of GPR should be also compared with other techniques, as manipulative therapy and cognitive behavioural therapy.

\section{References}

1. Henchoz Y, Kai-Lik So A (2008) Exercise and nonspecific low back pain: a literature review. Joint Bone Spine 75:533-539

2. Vanti C, Generali A, Ferrari S, Nava T, Tosarelli D, Pillastrini P (2007) Rééducation Posturale Globale in musculoskeletal diseases: scientific evidence and clinical practice. Reumatismo 59:192-201

\section{SPINE 2}

\section{Percutaneous axial lumbosacral interbody fusion: preliminary clinical and radiological results}

\author{
A. Ruggiu, A. Zachos, F. Barca, P. Tranquilli-Leali, F. Muresu, \\ C. Doria
}

Orthopaedic Department, University of Sassari (Sassari, ITALY)

Background Minimally invasive spine surgery continues to be a growing trend for orthopaedic and neurosurgical spinal interventions. Generally, anterior and posterior approaches are chosen for direct exposure of the lumbo-sacral spine. Anterior access to the L5S1 disc space for interbody fusion can be technically challenging, frequently requiring the use of a surgical approach for adequate exposure. We reviewed our experience with a novel minimally invasive technique for L5-S1 interbody fusion (Trans1) that exploits the pre-sacral space and its relative dearth of critical structures [1-3].

Material and methods Eight patients were included in this analysis. Average follow-up was 6 months. Back pain was secondary to lumbar degenerative disc disease (DDD) in 2 cases, failed-back surgery syndrome in 1 case and lytic spondylolistesis in another case. All patients had radiographic evidence of L5-S1 degeneration and underwent percutaneous fusion with Trans1 cage and local bone autograft. Trans1 was followed by percutaneous pedicle screw-rod fixation in 2 patients; in the remaining patients facet joint screw fixation devices were implanted. Clinical evaluation was performed using a visual analogue scale (VAS) and Oswestry form.

Results Mean operative time for the Trans1 procedure was $55 \mathrm{~min}$. All patients had radiographic evidence of stable L5-S1 interbody cage placement and fusion at last follow-up (Fig. 1). The VAS scores assessing back pain improved significantly from 7.20 to 2.65 . The mean Oswestry score improved significantly from 58.3 to $31.5 \%$. No device related complications were identified.

Conclusions The percutaneous paracoccygeal approach to the L5-S1 interspace provides a minimally invasive corridor through which discectomy and interbody fusion can safely be performed. It can be used alone or in combination with minimally invasive or traditional open fusion procedures (Fig. 1). It may provide an alternative route of access to the L5-S1 interspace in those patients who may have unfavourable anatomy for or contraindication to traditional open anterior approach to this level. 


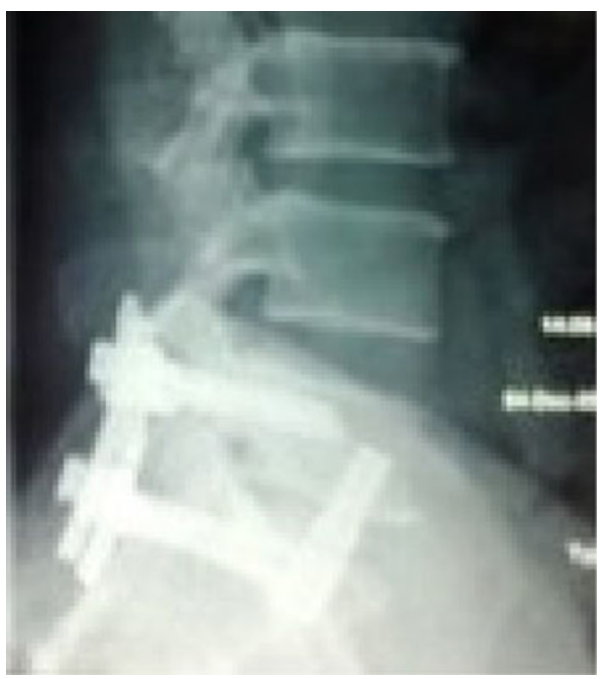

Fig. 1 L5-S1 interbody cage placement and fusion

\section{References}

1. Marotta N, Cosar M, Pimenta L et al. (2006) A novel minimally invasive presacral approach and instrumentation technique for anterior L5-S1 intervertebral discectomy and fusion: technical description and case presentations. Neurosurg Focus 20:E9

2. Cragg A, Carl A, Casteneda F et al. (2004) New percutaneous access method for minimally invasive anterior lumbosacral surgery. J Spinal Disord Tech 17:21-28

3. Muhlbauer M, Pfisterer W, Eyb R et al. (2000) Minimally invasive retroperitoneal approach for lumbar corpectomy and anterior reconstruction. Technical note. J Neurosurg 93[Suppl $1]: 161-167$

\section{Interspinous process decompression with the BacJac device for lumbar spinal stenosis: preliminary clinical experience}

\author{
A. Zachos, A. Ruggiu, C. Doria, F. Barca, F. Muresu, \\ P. Tranquilli Leali
}

Orthopaedic Department, University of Sassari (Sassari, ITALY)

Background Lumbar spinal stenosis is defined as the narrowing of either the spinal canal or neural foramina [1]. Underlying causes for the stenosis are hypertrophy of the ligament flavum and facet joints, osteophytes, spondylolisthesis, disc protrusion. The BacJac is a new interspinous process decompression device designed to distract the posterior elements of a stenotic lumbar segment and place it in flexion in those patients with neurogenic intermittent claudication [2, 3].

Material and methods We present 5 months follow-up data on the BacJac patients. The inclusion criteria for the trial were leg, buttock, with back pain relieved during flexion and claudication. The exclusion criteria were fixed motor deficit, cauda equina syndrome, previous lumbar surgery or spondylolisthesis greater than grade I at the affected level. From April 2008 to January 2010, thirty-eight patients were included in this study. The average age was 57 years. Twenty-six patients had the BacJac implanted at either L3-L4, L4-L5 or L5-S1 levels. Ten patients had the BacJac implanted at both L3-14 and L4-L5 levels and two patients at three levels L2-L3, L3-L4 and L4-L5. Two patients had a grade I spondylolisthesis, the other seventeen single or multi level spinal degenerative stenosis.

Results The mean pre-operative Oswestry score was 47 . The mean post-operative Oswestry score was 16 . The mean pre-operative VAS score was 8.7 and the mean post-operative one was 3.8. Our results demonstrated a very good success rate in the BacJac interspinous process decompression group at an average of 5 months postoperatively.

Conclusions The initial effectiveness data as seen in the significant improvements on both VAS and ODI also suggested that the BacJac (Fig. 1) could be a viable treatment option for patients with low-back pain caused by degenerative disc disease and low grade spondylolisthesis. Furthermore, the characteristic feature of this device is the low invasiveness and the possibility of use it in L5-S1 thanks to the variety of sizes and its simple surgical approach.

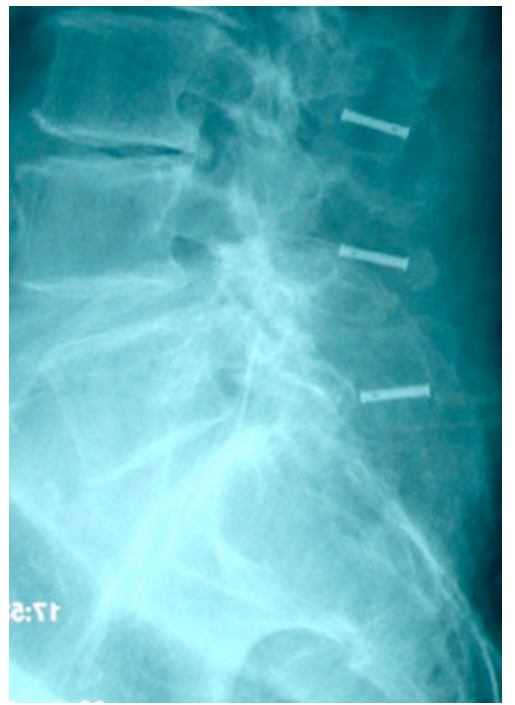

Fig. 1 BacJac device

\section{References}

1. Herno A, Saari T, Suomalainen O, Airaksinen O (1999) The degree of decompressive relief and its relation to clinical outcome in patients undergoing surgery for lumbar spinal stenosis. Spine 24:1010-1014

2. Schnake KJ, Putzier M, Haas NP, Kandziora F (2006) Mechanical concepts for disc regeneration. European Spine Journal, 15[Suppl 3]:S354-S360

3. Oppenheimer JH, DeCastro I, McDonnell DE (2009) Minimally invasive spine technology and minimally invasive spine surgery: a historical review. Neurosurg Focus 27(3):E9 